\title{
Artelogie
}

Recherche sur les arts, le patrimoine et la littérature de l'Amérique latine

$6 \mid 2014$

Horizons et dispositifs des arts plastiques des pays du Río de la Plata (XXe siècle)

\section{De Cuneo à Perinetti. Influences de l'art européen en Amérique du sud : la réplique d'un peintre uruguayen}

Régine Cardis

\section{(2) OpenEdition}

Journals

Édition électronique

URL : http://journals.openedition.org/artelogie/1241

DOI : 10.4000/artelogie.1241

ISSN : 2115-6395

Éditeur

Association ESCAL

Référence électronique

Régine Cardis, «De Cuneo à Perinetti. Influences de l'art européen en Amérique du sud : la réplique d'un peintre uruguayen », Artelogie [En ligne], 6 | 2014, mis en ligne le 24 juin 2015, consulté le 01 mai 2019. URL : http://journals.openedition.org/artelogie/1241 ; DOI : 10.4000/artelogie.1241

Ce document a été généré automatiquement le 1 mai 2019

Association ESCAL 


\title{
De Cuneo à Perinetti. Influences de l'art européen en Amérique du sud : la réplique d'un peintre uruguayen
}

\author{
Régine Cardis
}

1 Peintre figuratif jusqu'en 1956, interprète original du campo uruguayen, son thème de prédilection pendant plus de quarante ans, José Cuneo (1887-1977) se lance à l'âge de soixante-dix ans dans la peinture abstraite sous le nom de Perinetti. Cette conversion inattendue et brutale s'est imposée au peintre entre 1954 et 1956 lors du séjour en Europe qu'il effectue en usufruit de la bourse accordée par l'État uruguayen au lauréat de la première Biennale nationale d'arts plastiques, séjour pendant lequel l'artiste va et vient entre la France et l'Italie, la Hollande et l'Espagne ${ }^{1}$. Jusqu'alors, son intérêt pour l'art abstrait s'était limité à la lecture d'un certain nombre d'ouvrages théoriques parvenus en Uruguay après la guerre. À Amsterdam, José Cuneo découvre les œuvres du groupe Cobra et constate que l'équilibre du plan orthogonal créé par Mondrian et prôné en Uruguay par Torres García n'est plus en vigueur, qu'il a été «pulvérisé » par des peintres comme Appel et Corneille 2 . Mais son approche de l'art abstrait est encore de nature strictement spéculative. Les paysages qu'il peint entre 1955 et 1956 aux alentours du lac d'Isée en Italie ne laissent en rien prévoir la future mutation de José Cuneo en J. C. Perinetti, et ce n'est qu'à son retour à Montevideo, en 1957, que l'artiste uruguayen peint ses premières œuvres abstraites. La conférence qu'il donne en 1958 est un historique de l'art abstrait, dont il attribue l'origine à Cézanne, et qui apparaît comme la justification du changement radical qui vient de s'opérer dans sa propre peinture, changement pour lui nécessaire mais dont il ne définit pas clairement l'orientation ${ }^{3}$. Le bagage théorique et muséologique que l'artiste s'est forgé au cours de ses lectures et de ses voyages et qui est à l'origine de cette mutation, les difficultés qu'il dit avoir rencontrées pour assimiler ce nouveau langage semblent indiquer que le peintre n'est encore pas tout à fait prêt à l'assumer ${ }^{4}$.

2 José Cuneo a toujours cru au rôle catalyseur de l'art européen ${ }^{5}$. Conscient des carences dont souffre son pays en matière de collections d'œuvres d'art, il insiste, tout au long de sa vie, sur la nécessité de développer et d'améliorer le système de bourses d'études pour 
permettre aux artistes uruguayens d'aller se former en Europe. Lui-même n'y a guère effectué de séjours d'une durée supérieure à plus d'un ou deux ans, et l'essentiel de son activité artistique s'est déroulé en Uruguay ${ }^{6}$. José Cuneo est resté partagé entre sa curiosité toujours en éveil à l'égard de la culture européenne - et française en particulier -, et son attachement à sa terre natale.

Entre son premier voyage en Italie en 1909 et son séjour à Paris en 1912, José Cuneo a fait l'expérience de la peinture de paysage au contact de la tradition italienne des macchiaioli et des « luministes » espagnols via Blanes Viale []. Le thème du paysage lui a été dicté par son premier maître, le peintre turinois Anton Mucchi, qui a exploré les Préalpes piémontaises et peint de nombreux paysages, en particulier dans la vallée de la Suse ${ }^{8}$. Selon Cuneo, Mucchi avait commencé à peindre en plein air, directement sur le motif, comme le faisaient les impressionnistes, mais conservait dans sa palette des tons sombres, marron et noirs, ce qui le rattachait encore à l'académisme du siècle passé ${ }^{\text {. }}$ Grâce à Mucchi, il apprend cependant à voir la couleur dans la nature, telle que l'ont révélée les impressionnistes. Un petit paysage du lac de Garde, daté de 1909, montre que le jeune peintre ne cherche pas encore à traduire la réalité sensorielle de la lumière, mais à donner de la nature une image vivante par la spontanéité de la touche et la générosité de la matière, à la manière des macchiaioli. La gamme colorée y est très sobre, comparée aux quelques autres paysages d'Italie que nous connaissons et dans lesquels la leçon de l'impressionnisme qui consiste à traduire la lumière par la couleur et à voir les ombres colorées a été assimilée (cf. ill. 1 et 2). Les ombres sont désormais bleues ou violettes. Associés aux divers tons de vert de la végétation ces tons froids vont devenir prédominants à partir de 1910 .

1. Paysage d'Italie, 1909, Montevideo, coll. part.

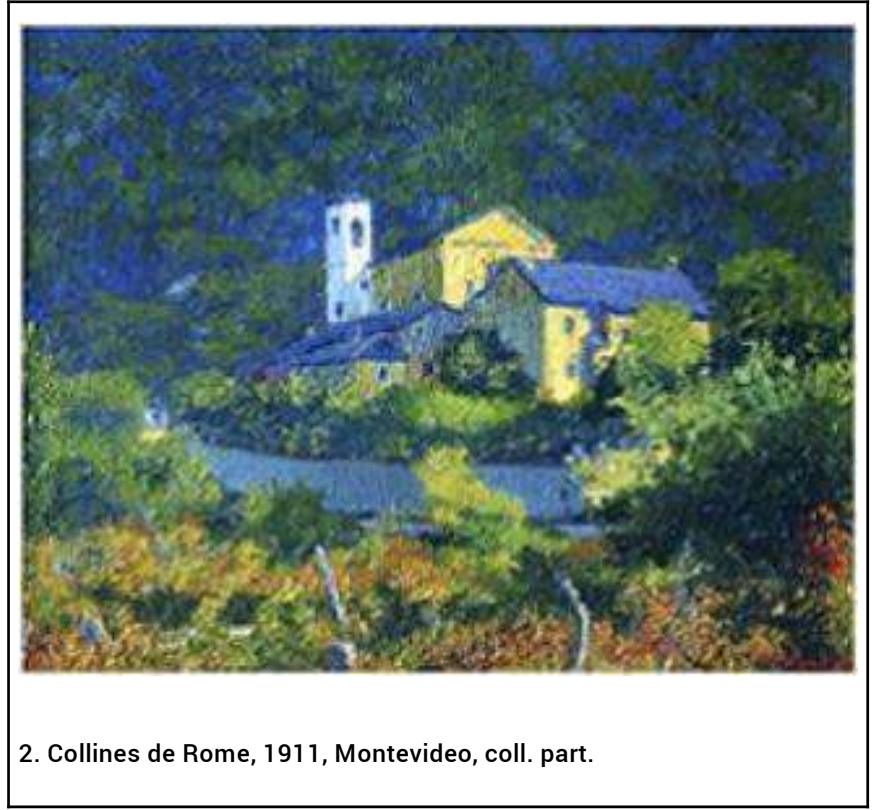




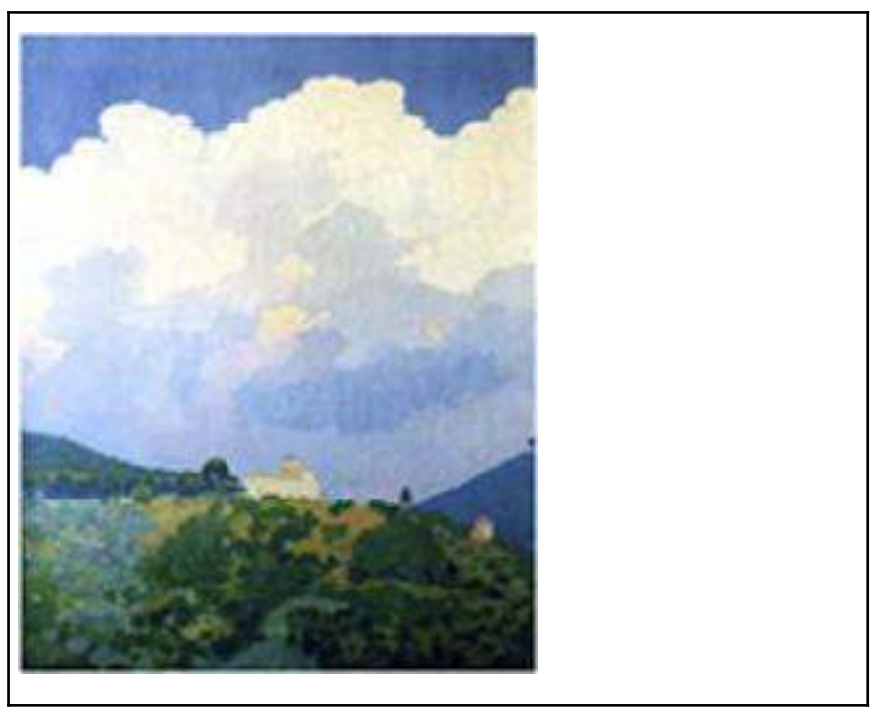

Les jardins des villas romaines que José Cuneo peint aux environs de Rome en 1911 à l'occasion de son deuxième séjour en Italie ne sont pas sans évoquer les vues du parc de Raixa à Mallorque, peintes par Blanes Viale en 1907, et l'on peut supposer que les vues des jardins de Saint-Cloud et du parc Monceau, peintes par Blanes Viale entre 1911 et 1914, ne sont pas inconnues de José Cuneo à l'époque où lui-même travaille, avec son ami Carmelo de Arzadun, dans les jardins du Luxembourg. Bien que n'ayant jamais entretenu de relations étroites avec son compatriote, de sept ans son aîné, le jeune Cuneo a été sensible à l'œuvre de cet artiste, réputé dans son pays et dans le milieu mallorquin. La thématique des parcs et des jardins, transmise aux peintres uruguayens par les peintres catalans Santiago Rusiñol (1861-1931) et Joaquim Mir (1873-1940), constitue à cette époque pour le jeune Cuneo un passage obligé.

1912, José Cuneo apprécie autant l'œuvre du peintre espagnol Anglada Camarassa que celle de Kees Van Dongen, ses deux professeurs à l'Académie Viti. La peinture fauve n'est pour lui qu'un « isme » de plus, et il s'écoulera un certain temps avant qu'il n'adopte un point de vue critique à l'égard d'Anglada, jugeant que celui-ci se rapproche des fauves par l'utilisation de couleurs violentes mais que sa peinture reste largement tributaire du réalisme espagnol du point de vue formel ${ }^{10}$.

Dans les paysages du département du Treinta y Tres, que José Cuneo peint en 1913, à son retour d'Europe, la couleur s'étend sur de larges surfaces et n'est pas fragmentée par la touche comme dans certains paysages des jardins du Luxembourg (cf. ill.3). Cette nouvelle manière de formuler le paysage par des aplats de couleurs pures et une certaine stylisation, que l'on observe notamment dans la façon dont le peintre décrit les ondulations du sol et la forme des pierres, témoigne de l'influence des œuvres des Nabis qu'il a pu voir à Paris, et n'est probablement pas étrangère non plus à l'enseignement d'Anglada. Les figuiers de barbarie, les caroubiers au feuillage en ombelle, les rochers bleus, les ruisseaux aux berges sinueuses qui se perdent dans la prairie, cèdent la place, à partir de 1918, à de grands arbres à la silhouette charpentée dont certains semblent avoir été découpés aux ciseaux, à de larges rivières dont les berges rectilignes s'étalent au premier plan et entrent dans le jeu orthogonal de la composition (cf. ill. 4 et 6). 
3. Entre las luces, 1916, Montevideo, Musée Blanes

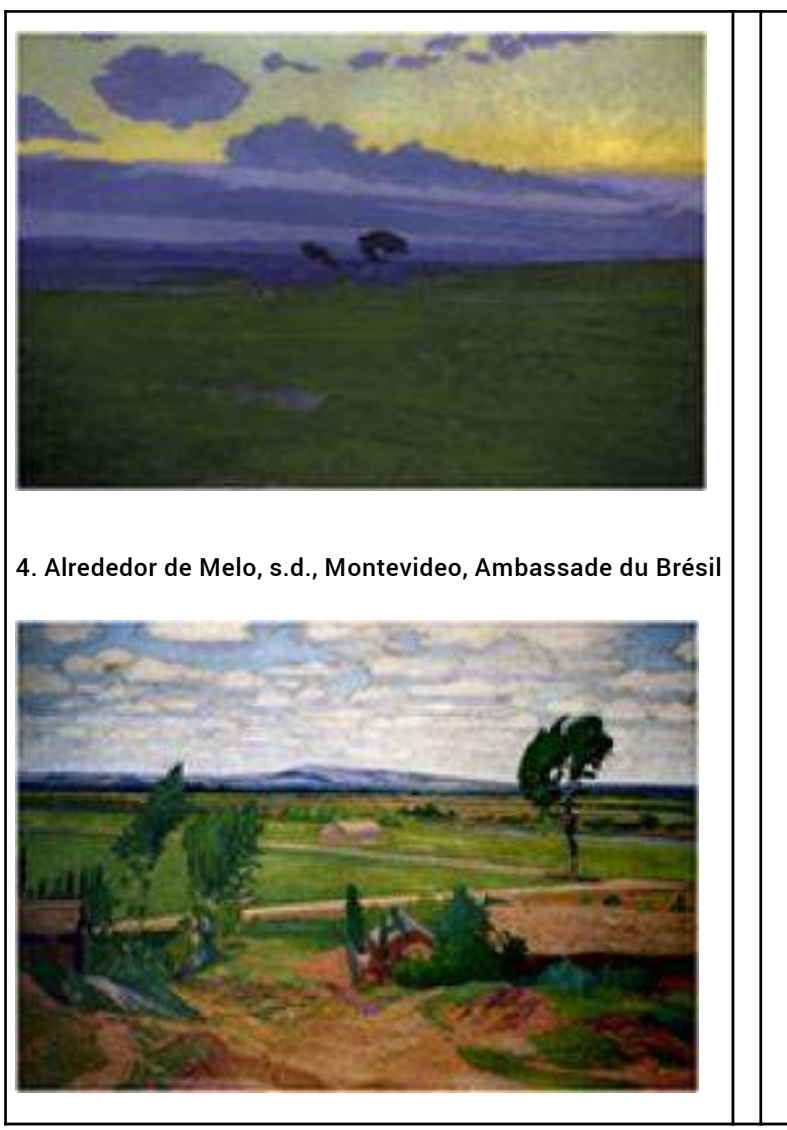


5. La famille Percivale, s.d., Montevideo, Musée national des Arts visuels (exemple de style " planiste » dans la peinture de portrait)

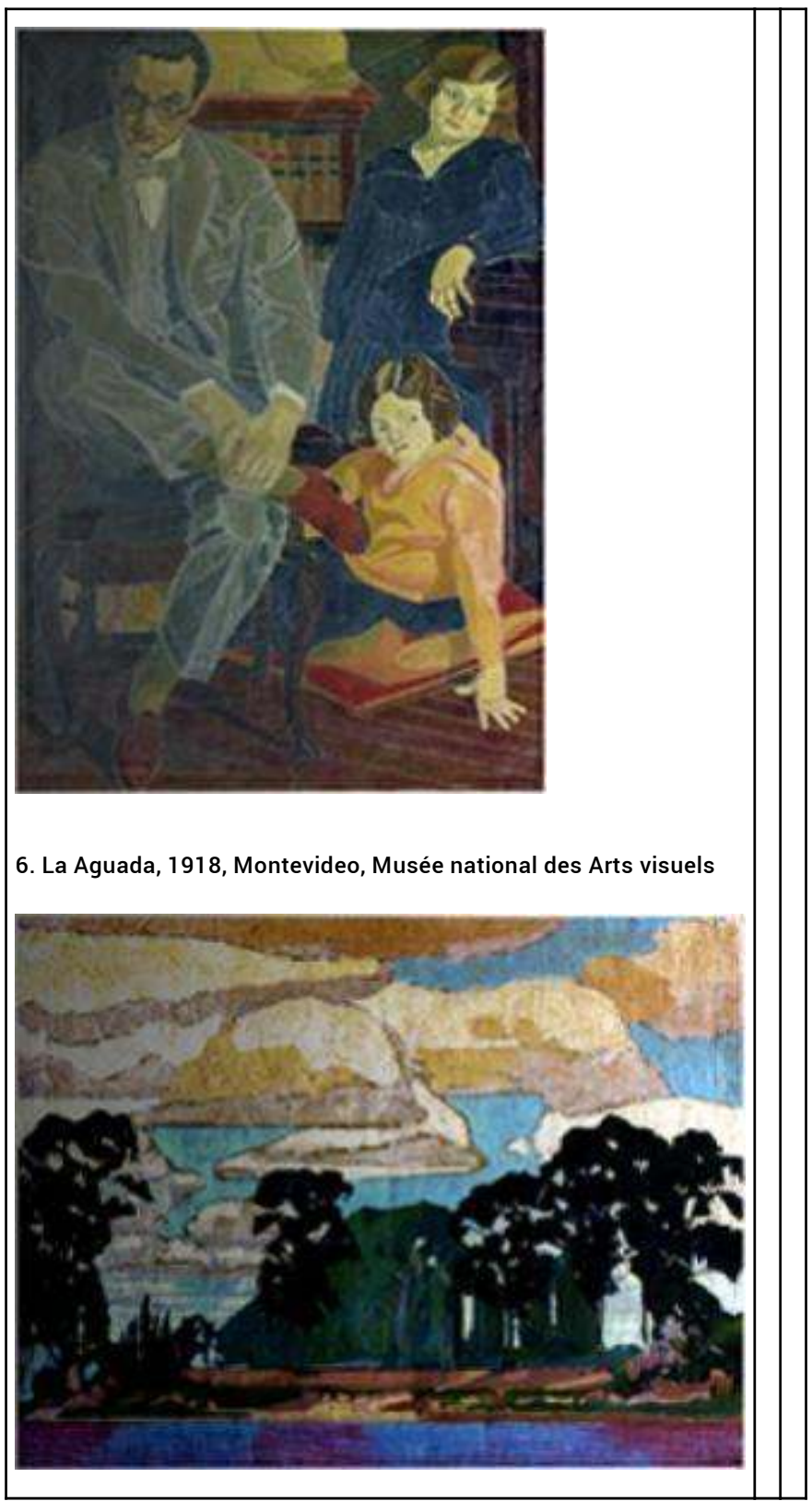

7 L'ordonnance quasiment architecturale que le peintre donne à ses paysages du Cerro Largo, leurs compositions stables, à dominante horizontale, l'aspect monolithique des arbres et des nuages, sont peut-être dus à une influence de l'œuvre de Cézanne, mais peuvent aussi être comparés à la solidification des volumes qui s'effectue à partir de 1908 dans les paysages fauves, phénomène qui a pour origine l'intérêt croissant manifesté par les fauves à l'égard de l'œuvre du maître d'Aix-en-Provence et pour conséquence le glissement d'un certain nombre d'entre eux vers le cubisme (Figari a reproché à Cuneo ses «tentatives cubistes »). L'aspect massif qui a compté pour ces artistes comme facteur déterminant dans le choix de certains motifs est caractéristique de la série des "îles ", peinte en 1922 par José Cuneo. En effet, ce qui semble avoir séduit le peintre dans le motif 
de l'«île», dont il fait le sujet principal d'une série de sept paysages, c'est son assise sereine et stable, et la régularité du schéma constructeur qu'il offre à la représentation ${ }^{11}$. Le découpage des éléments du paysage, que l'on a pu comparer à un puzzle, est cependant assez éloigné de la manière dont Cézanne suggère la forme par la juxtaposition de plans colorés. D'autre part, la volonté de l'artiste de simplifier les formes et de faire une peinture plane (d'où la dénomination de planismo), qui témoigne aussi de l'influence de l'œuvre de Gauguin, n'a pas toujours engendré un style synthétique. José Cuneo admet avoir créé parfois des effets de reliefs par le morcellement excessif des surfaces, ce qui est en complète contradiction avec l'esprit du planisme ${ }^{12}$. Seuls quelques paysages de Maldonado, peints entre 1918 et 1919, en particulier La Tour de la vigie ${ }^{13}$, sont composés de larges aplats synthétiques et de formes strictement imbriquées, ce qu'a facilité, il est vrai, la présence des éléments architecturaux. L'utilisation de couleurs pures, «sortant du tube » comme se plaisait à le dire José Cuneo, a pu être interprétée comme une influence de la peinture fauve avec laquelle celui-ci s'y est familiarisé à travers l'enseignement de Van Dongen et les expositions qu'il a vues à Paris, mais la couleur conserve chez lui une fonction descriptive. Cuneo et les artistes de sa génération restent fidèles tant du point de vue de la forme que de la couleur à ce que Dieste appelle «l'ordre plastique naturel ${ }^{14}$ ». Chercher à traduire la «lumière locale » implique, dans le contexte idéologique des années vingt, un rapport affectif voire sentimental avec le paysage natif, qui transparaît dans le chromatisme des œuvres. Le jeu des contrastes colorés se maintient dans une douce harmonie. L'atmosphère d'intense sérénité qui se dégage d'un paysage tel qu' Alentours de Melo (Cerro Largo) (ill. 4) vient de l'équilibre stable de la composition tout autant que de l'emploi d'une gamme de couleurs tendres, d'une luminosité voilée.

La découverte de l'œuvre de Chaim Soutine à l'occasion d'un nouveau séjour en Europe, entre 1928 et 1930, a des conséquences très importantes sur l'évolution de l'œuvre de José Cuneo $^{15}$. L'artiste prend en compte certains aspects novateurs de la peinture européenne qu'il avait jusqu'alors négligés, en particulier l'expression du mouvement mais aussi la libre interprétation du monde visible. Cependant, la volonté régulatrice qui s'est affirmée dans l'œuvre planiste de José Cuneo pendant les années vingt n'a pas cédé au contact de l'impulsion expressive et passionnée de Soutine. Cette impulsion est à l'origine de la distorsion que ce dernier impose à ses paysages, ses natures mortes et ses portraits. En revanche, l'impression de mouvement et de vacillation du paysage que José Cuneo crée dans certaines œuvres peintes à Cagnes-sur-Mer est rigoureusement étudiée; elle n'est pas imputable au tempérament du peintre. Cuneo emprunte à l'œuvre de Soutine un certain nombre d'a priori formels, qu'il emploie, certes, de manière emphatique et démonstrative (recours systématique aux diagonales, organisation de l'espace autour d'un centre qui n'est pas le point de fuite traditionnel de la perspective) (cf. ill. 7 et 8), mais qu'il saura intégrer, dans la série des « Ranchos y Lunas », à une vision personnelle et originale du paysage natif. 
7. Paysage de Cagnes-sur-Mer, 1928-29, loc. inc.

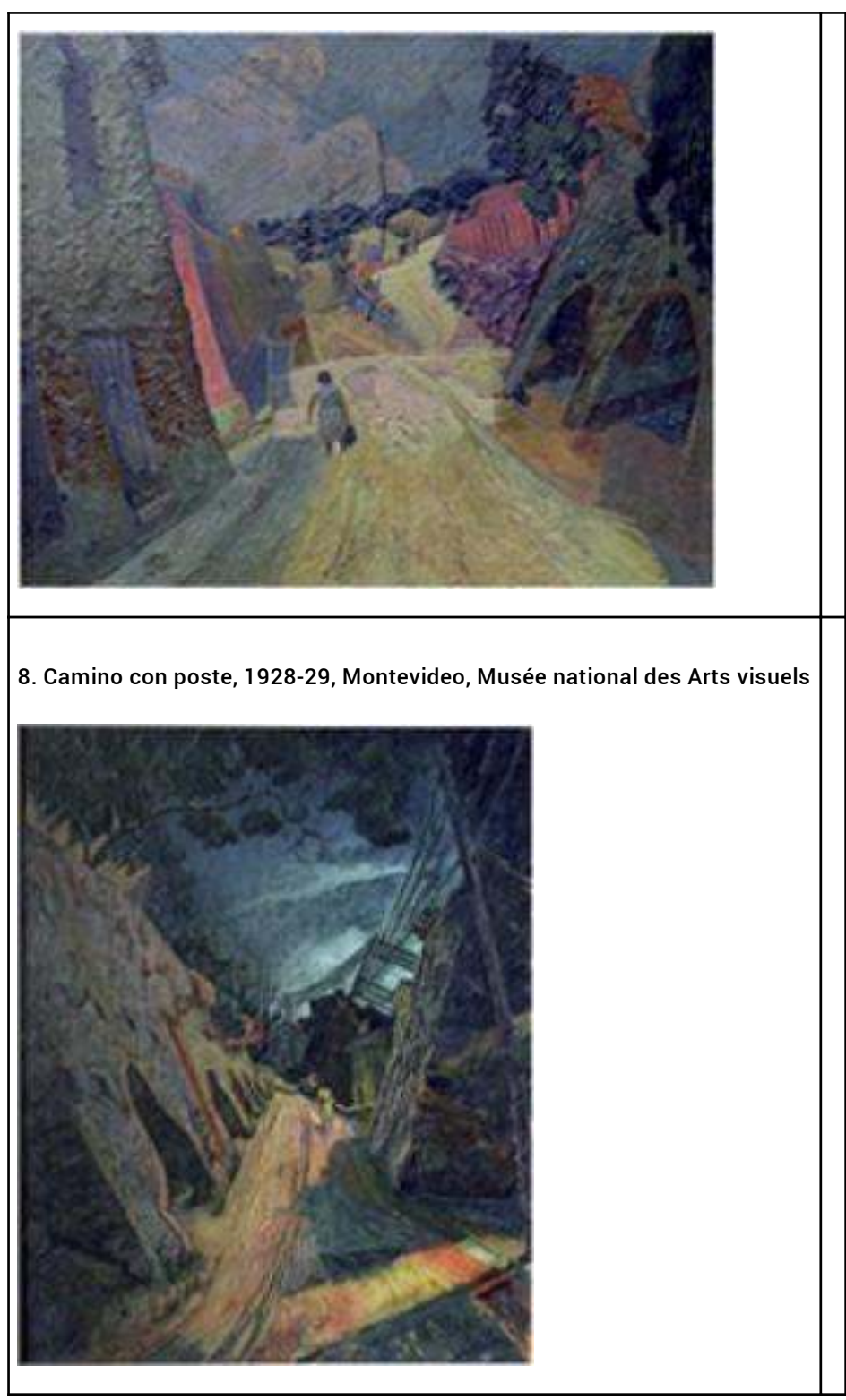


9. Paysage de Cagnes-sur-Mer, 1928-29, Montevideo, coll. part.

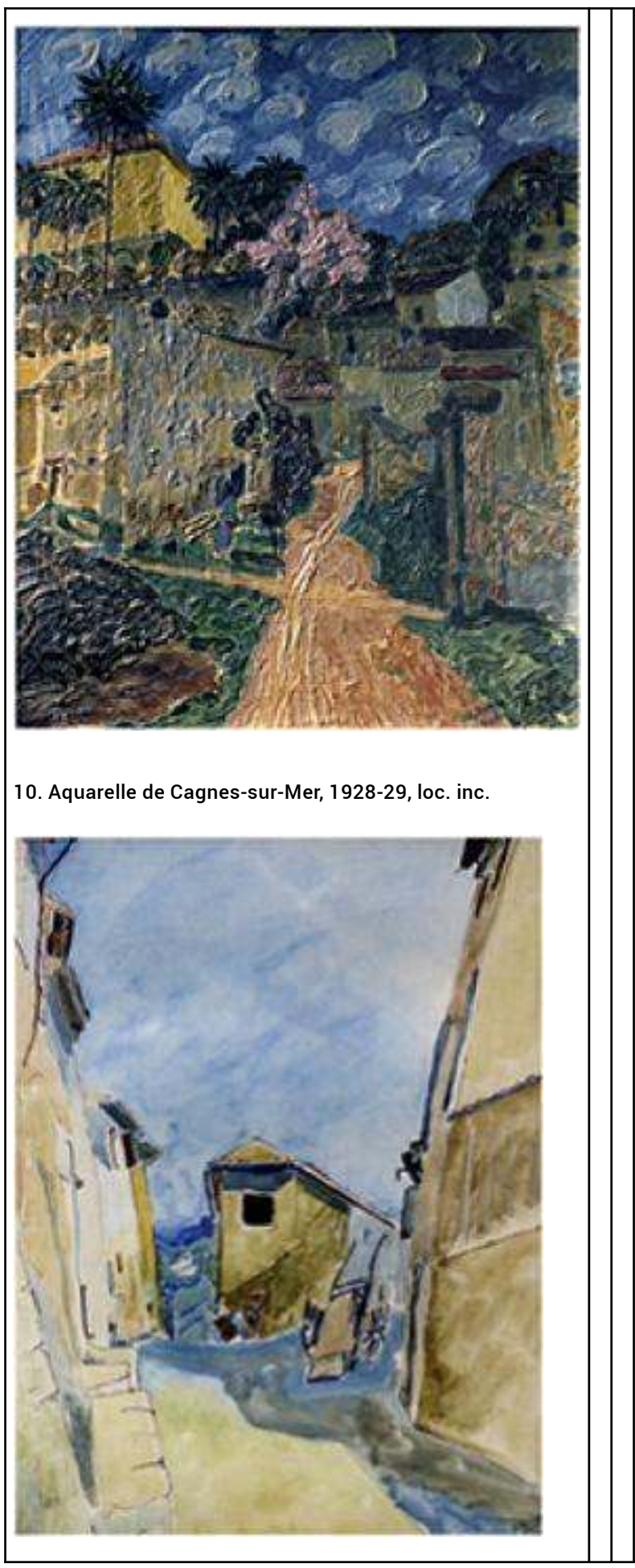

9 À la même époque, José Cuneo redécouvre l'œuvre de Cézanne lors de ses conversations avec le peintre d'origine égyptienne Diran Garabedian (1882-1963), qui fut l'un des amis les plus proches du peintre uruguayen pendant son séjour à Cagnes. Comme il le dira luimême de nombreuses années plus tard, ses paysages peints en Uruguay dans les années vingt ne conservent de l'exemple du maître d'Aix qu'une superficielle géométrisation ${ }^{16}$. 
En revanche, dans les paysages de Cagnes et dans la série des « Ranchos y Lunas », qu'il peint à son retour en Uruguay (cf. ill. 11 à 15), les déformations optiques auxquelles Cuneo soumet la représentation du paysage afin d'adapter ses différents éléments à la surface plane du fond signalent un degré supérieur d'interprétation de la spatialité de Cézanne, spatialité sous-jacente dans l'œuvre de Soutine. Mais tandis que chez Soutine la compression de l'espace engendre peu à peu une fusion des formes et de la matière, elle devient, chez Cuneo, l'instrument d'une vision totalisante dans laquelle les éléments terrestres et célestes du paysage sont absorbés par un mouvement qui semble déborder des limites de la toile et, en même temps, sont attirés vers son centre comme par une force magnétique. L'espace se concentre et se dilate ; comme l'a dit Cipriano Vitureira, il est conçu en fonction d'un cadre mais multiplié à l'échelle de l'univers ${ }^{17}$. Le phénomène d'inversion de la perspective, taxé de "perversion esthétique » par les rédacteurs de la revue Removedor (organe de presse du Taller Torres-García entre 1945 et 1953), relève de cette volonté même d'intégrer les objets dans un espace que l'œil peut embrasser dans sa totalité. Le cubisme et son principe de vision simultanée se sont infiltrés dans l'œuvre de Cuneo par des voies détournées. Les théories d'André Lhote, diffusées à partir de 1932 à Montevideo par Carlos Prevosti à l'Escuela Taller de Artes Plásticas ${ }^{18}$, ont aussi contribué à consolider dans l'œuvre de Cuneo une conception de l'espace fondée sur «l'utilisation intégrale de la surface sans vides inexpressifs ${ }^{19}{ }$.

11. Ranchos entre eucaliptus, 1931, Montevideo, coll. part.

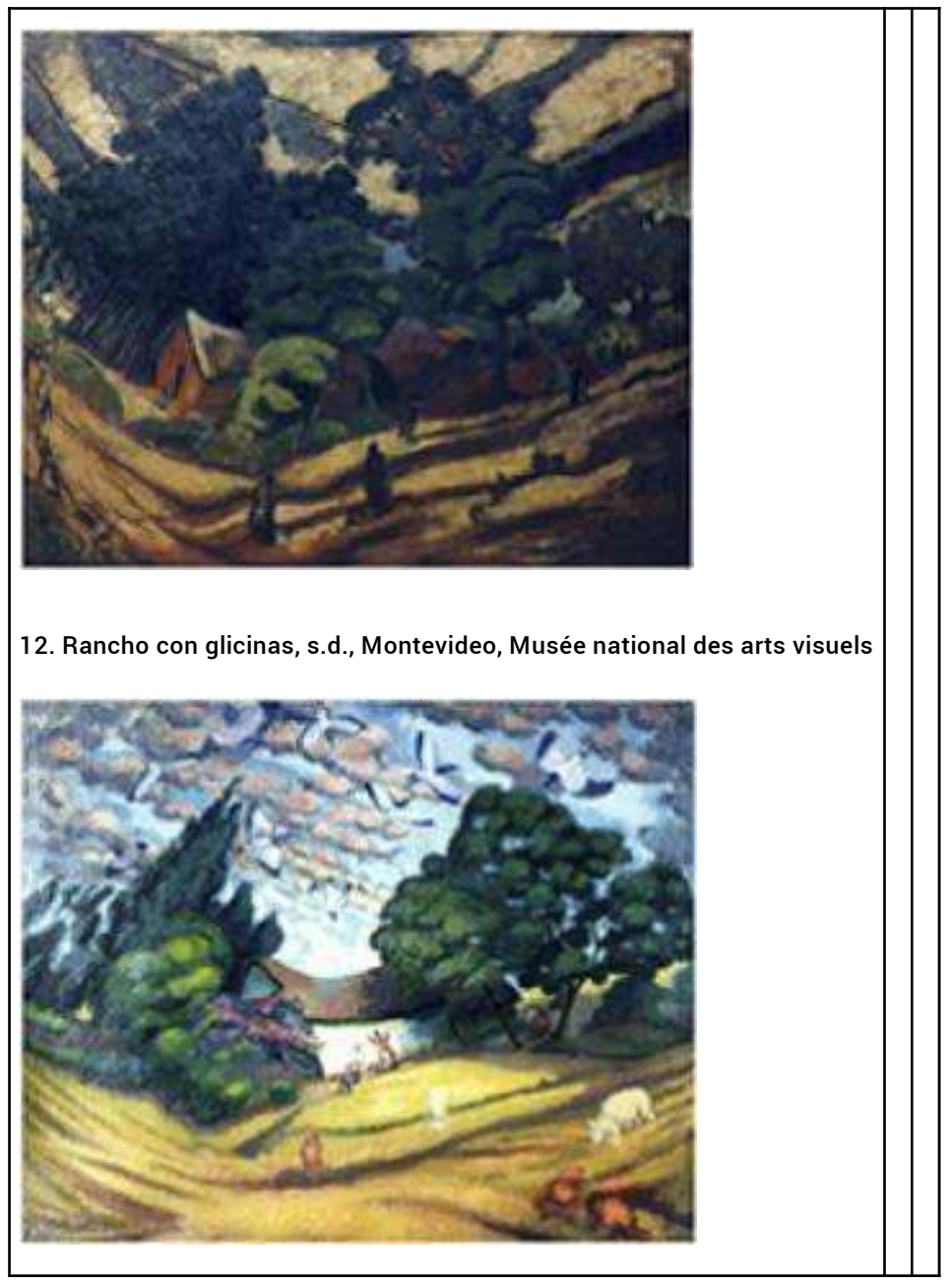


13. Suburbios de Florida, s.d., Montevideo, Musée national des Arts visuels ;

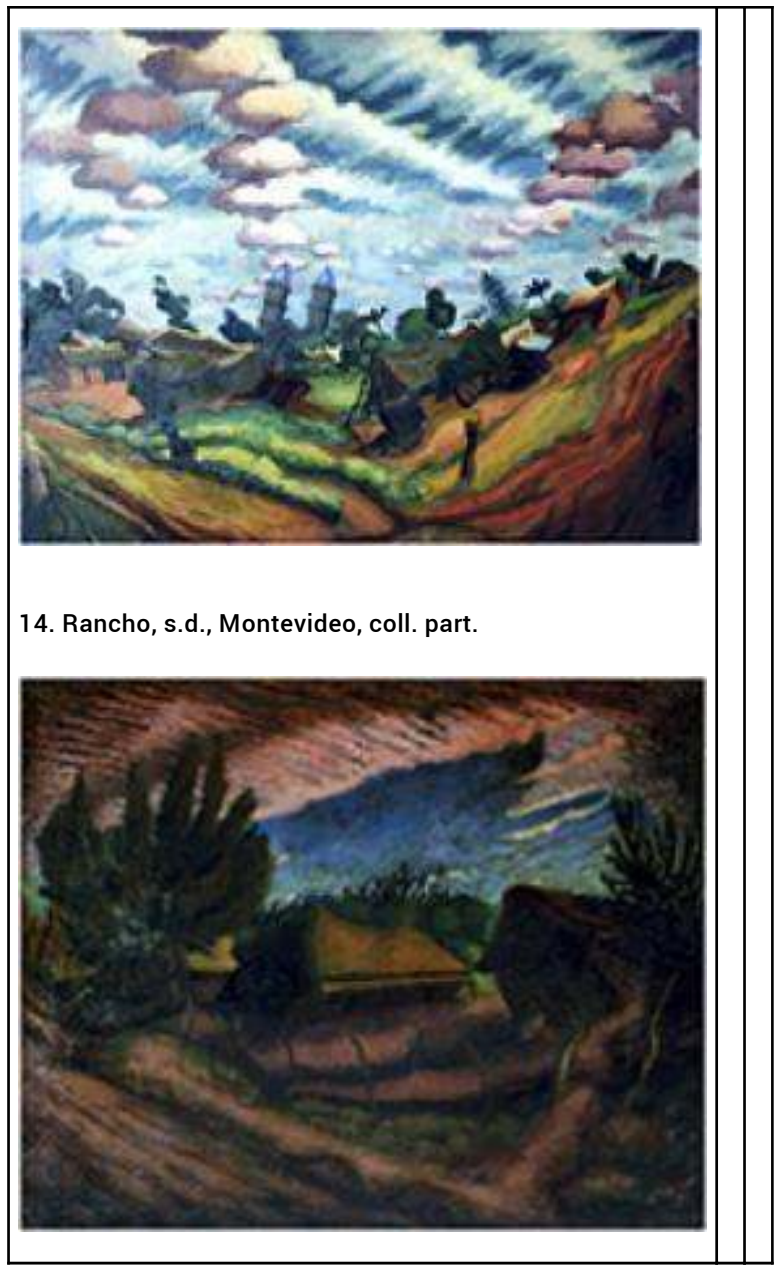

15. Rancho de Florida, ca 1934, Montevideo, Palacio Legislativo

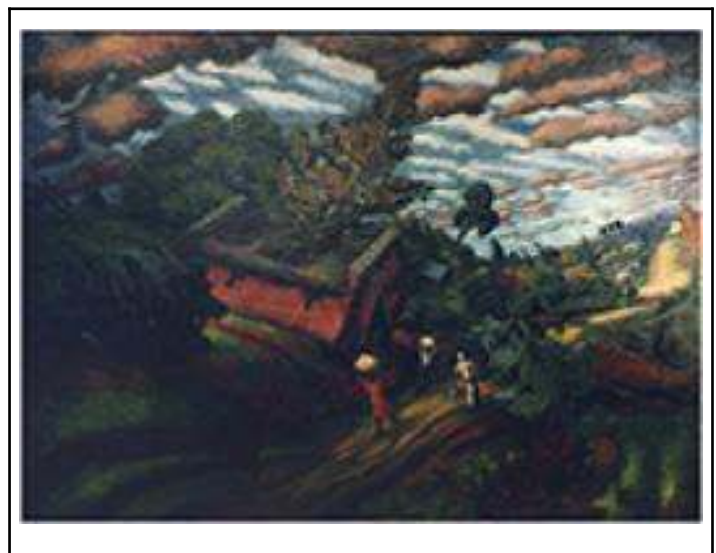

Cette conception spatiale est cependant filtrée dans la série des lunes (cf. ill. 16 à 28) au travers d'une représentation qui tend à la fois à respecter les données identifiables du paysage natif et à dévier la perception objective de ses différents motifs en les soumettant à un effet surnaturel (dilatation, démultiplication de la lune, paysage déformé sur la 
surface courbe de l'astre comme une image réfléchie par un miroir convexe) ou en les dotant d'une fonction symbolique (dialogue planétaire symbolisé par le face-à-face entre la lune et un motif terrestre - ranch, charrette, cactus, vache morte ou ravin -, fonction unificatrice des engoulevents, du motif de l'arbre et du poteau téléphonique, sortes de pont qui relie la terre et le ciel). Comme Figari, Cuneo joue parfois à forcer les apparences naturelles; il extrait des masses nuageuses des formes animales, réelles ou imaginaires. Bien que l'espace céleste des paysages cunéens respecte la topographie réelle des cieux uruguayens, les métaphores qui s'y opèrent relèvent d'une impulsion imaginative ainsi que d'une expression ludique voisine du surréalisme. 
16. Lune de l'aveugle, s.d., Montevideo, coll. part.

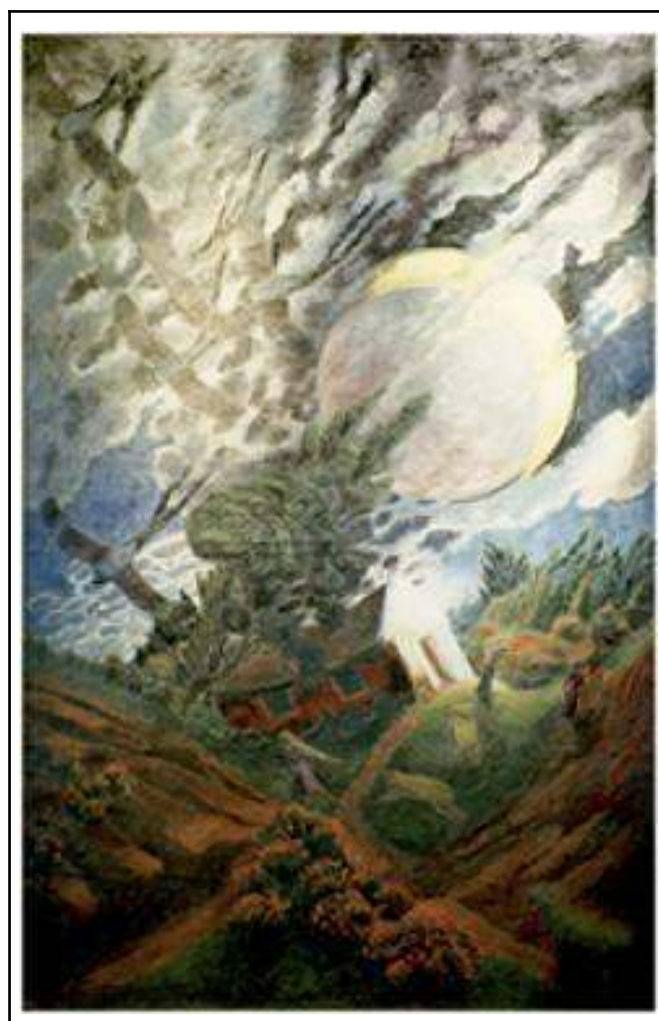

17. Cerco de tunas, s.d., Montevideo, Musée national des Arts visuels

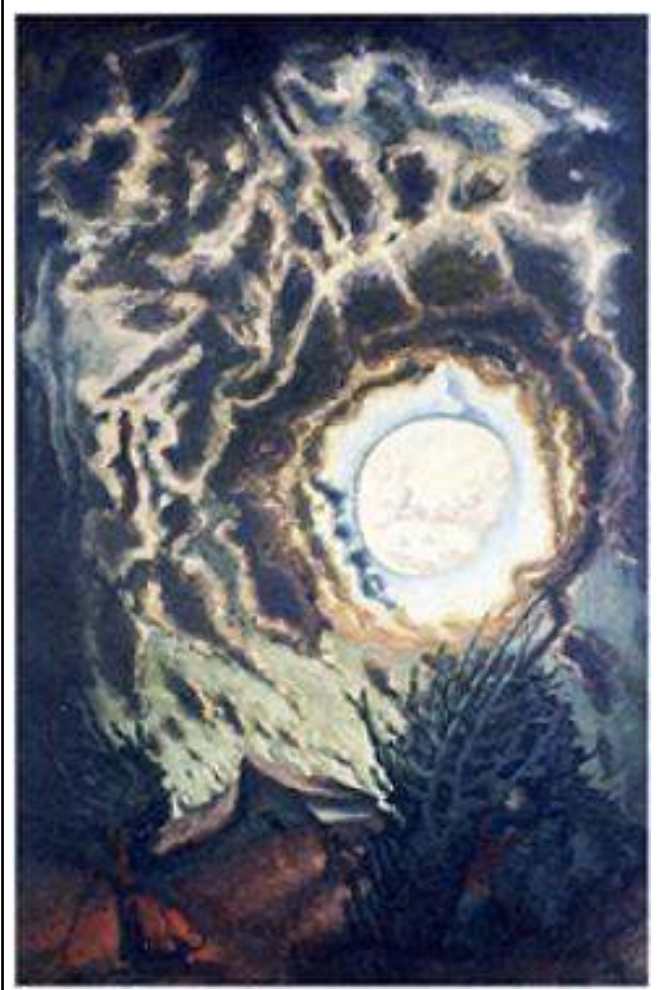




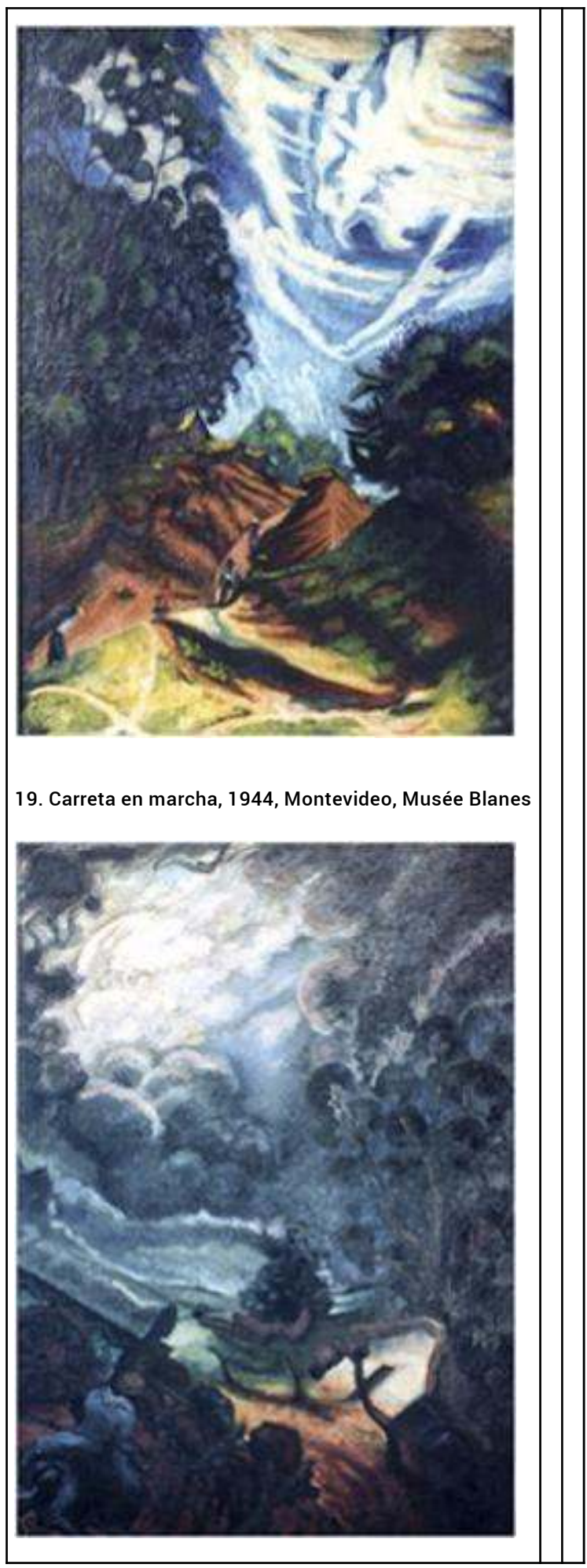

11 Mais l'un des aspects les plus surprenants et aussi les plus originaux de la série est le traitement du ciel, qui n'est ni un espace atmosphérique ni un écran neutre. José Cuneo part de l'observation directe du ciel, décrit les différents types de nuages et en montre la stratigraphie pour créer un espace doté d'une structure, d'une densité et d'une 
organisation qui lui sont propres. La déformation des éléments identifiables du paysage, d'une part, et, d'autre part, la consolidation de ses éléments diffus et mouvants afin de créer un espace autonome, situent dès cette époque le langage plastique de Cuneo aux confins du figuratif et de l'abstrait. Lune de l'herborisatrice (1931) ${ }^{20}$ contient déjà le vocabulaire des formes de l'époque Perinetti. Le réseau obscur de formes tentaculaires qui s'étend sur l'espace de la toile annonce des œuvres comme Lune noire (1962), Mains pattes (1961) et Oiseau (1961) ${ }^{21}$.

12 L'évolution de l'œuvre de José Cuneo après Cagnes-sur-Mer répond à la fois à des préoccupations formelles héritées de la peinture européenne et à une façon d'aborder métaphoriquement le paysage natif en accord avec la conception que son milieu culturel d'origine a de la relation entre l'homme et son environnement. Conception mentale du paysage imprégnée d'imaginaire "nativiste » et d'idéologie figarienne, laquelle préconise un retour aux sources et envisage le paysage américain comme lieu de cohérence originelle ${ }^{22}$. "L'utopie anthropologique » de Pedro Figari, qui consiste à retrouver une supposée unité perdue, est développée dans son œuvre littéraire mais aussi, comme l'explique Gabriel Peluffo Linari, dans son œuvre peint, œuvre dont la charge critique teintée d'humour tranche avec l'optimisme au travers duquel s'exprime la ferveur nativiste chez la plupart des peintres uruguayens pendant les années vingt ${ }^{23}$. Il est très probable que José Cuneo, qui faisait partie du cercle des figaristes dévots liés à l'association Teseo et à la revue Cruz del Sur, ait souhaité réaliser avec la série des «Ranchs et des Lunes » une œuvre capable de sublimer certains aspects du paysage local et d'offrir à son tour le spectacle de cette unité perdue en créant un lieu de correspondances entre le terrestre et le céleste. L'œuvre de José Cuneo et celle de Pedro Figari sont liées par certaines affinités idéologiques et certains rapports symboliques déchiffrables sur l'ensemble de la série, et ceci bien que les deux artistes s'opposent tant du point du vue du choix thématique, du style, que de la personnalité ${ }^{24}$.

13 Les Lunes de Cuneo révèlent une conception baroque de la relation entre le terrestre et le céleste $^{25}$. La distorsion des formes, le dynamisme de la composition, le recours systématique aux diagonales ont été interprétés comme une influence de Soutine. Ces mêmes facteurs stylistiques, ajoutés à une prédilection pour la pénombre et un certain climat irrationnel créé par la lumière, ont été mis au compte de l'intérêt que Cuneo portait à l'œuvre du Greco (dont Soutine était lui aussi un grand admirateur). Or ce serait négliger le contenu spirituel des Lunes que d'y voir une simple coïncidence formelle avec les œuvres du Greco. Dans les Lunes, la déformation du paysage, la dilatation de la lune, l'omniprésence du ciel se rapprochent de la vision du Greco chez qui « l'allongement des formes pourrait être l'expression artistique de la tension oppositionnelle entre la terre et le ciel dont les saints élongés sont une métaphore» (DANTO, 1993, p. 54). Le Greco est, avec Cézanne, le peintre à l'égard duquel Cuneo a manifesté la plus grande admiration. Il admire avant tout les aspects novateurs de sa peinture en matière de représentation de l'espace, l'équilibre dans la distribution des contrastes de valeur qui permet de rétablir sur le plan de la toile tous les éléments de la composition. 


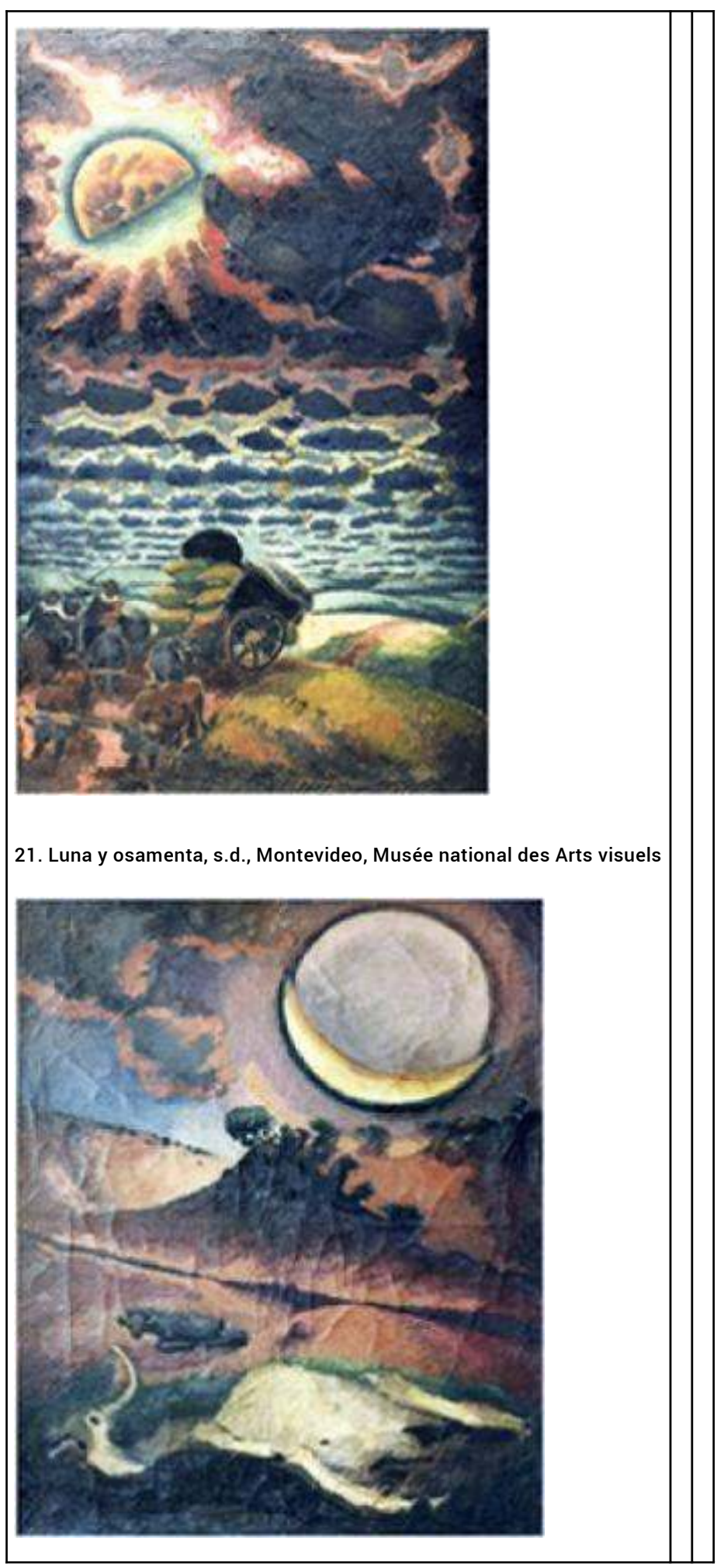

C'est en ce sens qu'il compare les derniers paysages de Cézanne (série de la montagne Sainte-Victoire et le Château noir) à la Vue de Tolède où, dit-il, le peintre parvient à donner l'illusion de semi-frontalité ${ }^{26}$. Ce n'est que tardivement, probablement dans les années 
soixante-dix, que José Cuneo a pu voir l'œuvre du Metropolitan Museum de New York, mais il a découvert la peinture du Greco bien avant cette époque, à l'occasion de ses nombreuses visites dans les musées européens, et a même entrepris la rédaction d'une étude sur certains tableaux du peintre espagnol vus à Rome à la Galerie Corsini ${ }^{27}$. Avant 1930, José Cuneo a lu des ouvrages sur le Greco, notamment ceux de Manuel B. Cossio et de J. F.Willumsen ${ }^{28}$. Son intérêt pour le Greco se révèle en particulier dans son article sur Rafael Barradas, publié à Montevideo en 1930. Il défend alors avec enthousiasme les œuvres d'inspiration religieuse, souvent controversées, du peintre uruguayen, affirmant que celui-ci y atteint un degré de mysticisme jamais égalé par un peintre espagnol depuis le Greco ${ }^{29}$. Le mysticisme de Barradas s'exprime, selon lui, dans l'élan affectif et poétique qui entraîne, comme chez le Greco, le triomphe de la lumière mais aussi la déformation des figures, le dynamisme de la composition, l'élasticité des formes et des volumes. Quelle que soit la validité de son argumentation, il est intéressant de constater qu'à cette époque les préoccupations esthétiques de José Cuneo vont dans le sens d'une interprétation spirituelle du monde autant que de la spécification intellectuelle du langage plastique. À la fin de son article, Cuneo laisse planer une interrogation qui est de savoir si un artiste uruguayen qui n'a pas été soumis comme Barradas à l'influence d'une culture fortement marquée par le catholicisme aurait pu traduire une telle inquiétude spirituelle ${ }^{30}$. La série des « Ranchos y Lunas », qu'il commence à peindre en cette même année 1930, est peutêtre la réponse qu'il souhaite donner à cette interrogation. 
22. Luna sobre la estancia, s.d., Musée de San José (Uruguay)

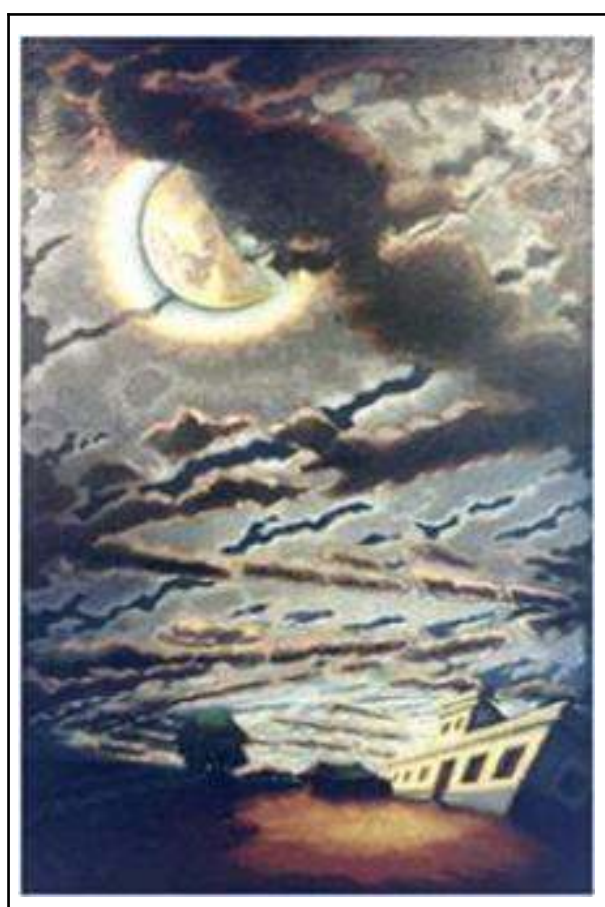

23. Luna sobre la estancia (détail)

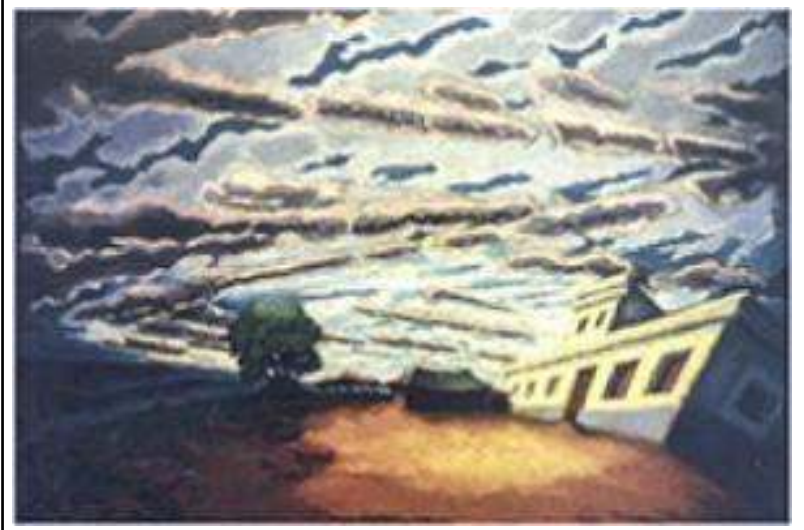

24. Luna en acegua, s.d., coll. part. 


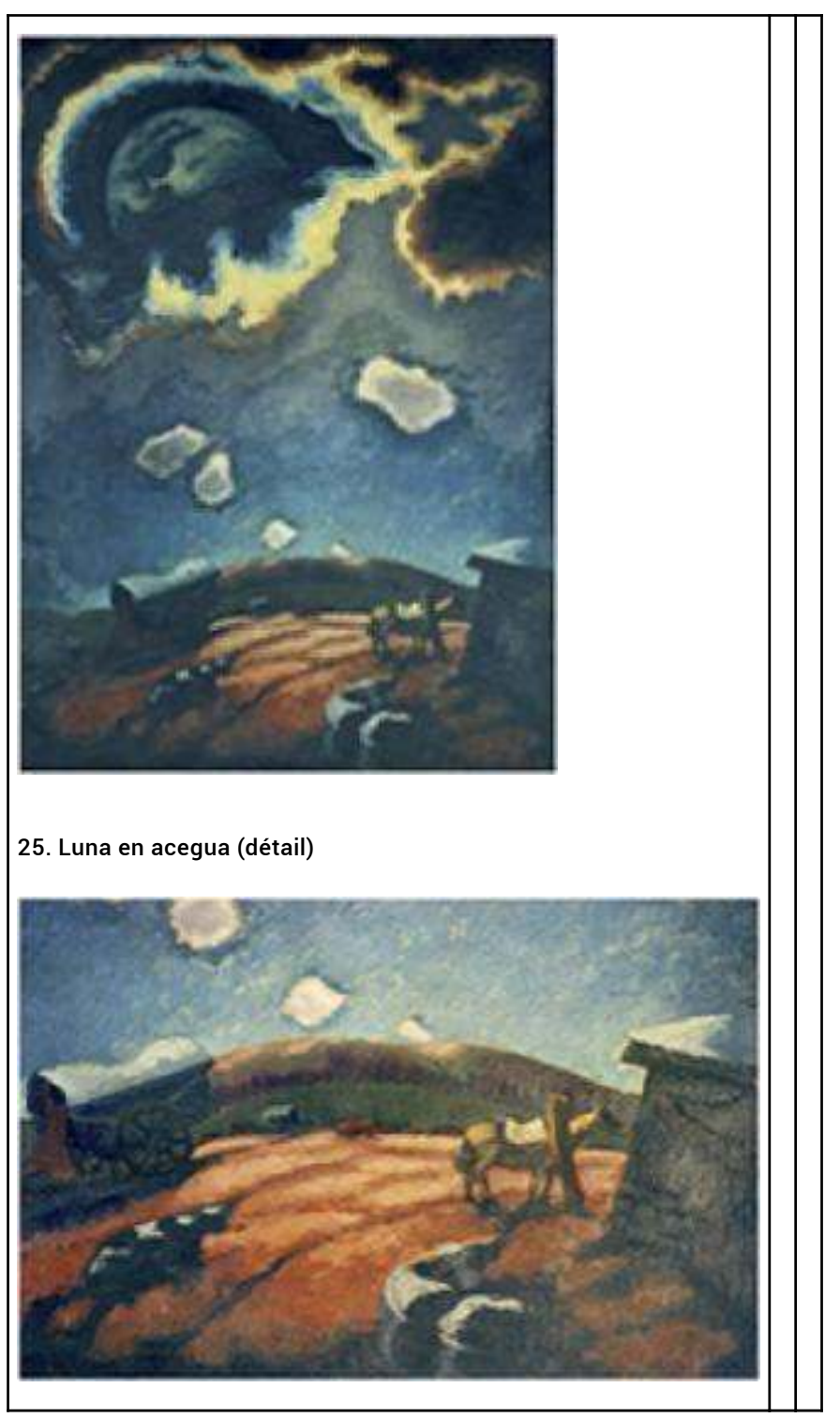

Nous avons mis en parallèle l'œuvre de José Cuneo et celle du Greco pour montrer une certaine affinité spirituelle dans leur conception de l'espace comme expression métaphysique des causes de l'univers. Mais une semblable inquiétude spirituelle se rencontre chez des peintres plus contemporains, dont un en particulier, pour lequel le peintre uruguayen a manifesté un grand intérêt, à savoir Paul Gauguin ${ }^{31}$. Si José Cuneo traite avec un certain mépris les peintres catholiques modernes comme Maurice Denis, dont il juge le spiritualisme artificiel et compassé comparé au mysticisme intuitif de Barradas $^{32}$, peut-être a-t-il été séduit par le symbolisme de l'œuvre de Gauguin. Rappelons qu'en 1917, à Paris, Cuneo a vu dans les réserves d'une galerie où il était venu assister au vernissage d'une exposition de son ancien maître Kees Van Dongen, le tableau de Gauguin D'où venons-nous? Que sommes-nous? Où allons-nous?, œuvre qui lui fit, dit-il, «une impression extraordinaire ${ }^{33} »$. En 1930, José Cuneo cite Octave Mirbeau pour parler du peintre français qui a fui la civilisation afin de « mieux écouter les voix intérieures qui se noient dans le tumulte de nos passions et de nos disputes ", et sa propre démarche reflète un sentiment équivalent face à la nature, au sens où, comme Gauguin, c'est au contact d'un paysage vierge redécouvert à son retour d'Europe, une première fois en 1913 et une 
seconde fois en 1930, que José Cuneo éprouve le sentiment du mystère de l'existence ${ }^{34}$. La dimension symbolique d'une œuvre comme Lune des trois pointes ${ }^{35}$ tient au climat d'humble recueillement qui se dégage du paysage, où le temps paraît suspendu à l'attente, celle du paysan sans visage assis face à son chien, et au geste immuable de la femme tirant de l'eau au puits qui nous tourne le dos, enfin, au caractère énigmatique de ce ciel nocturne, où la lune repose sur un appendice de nature indéfinissable formant avec l'auréole sombre qui contourne la face cachée de l'astre une sorte de point d'interrogation.

16 La charge symbolique dont est investi le paysage local dans le processus d'élaboration d'une identité nationale est tout aussi sensible dans l'œuvre paysagiste de José Cuneo postérieure à 1930 que dans ses paysages de l'époque planiste. Probablement conscient des limitations qu'imposait un semblable attachement à la fonction représentative de l'œuvre d'art, José Cuneo a préféré renoncer brutalement à tout support figuratif pour appréhender de façon abstraite l'espace et la couleur. 


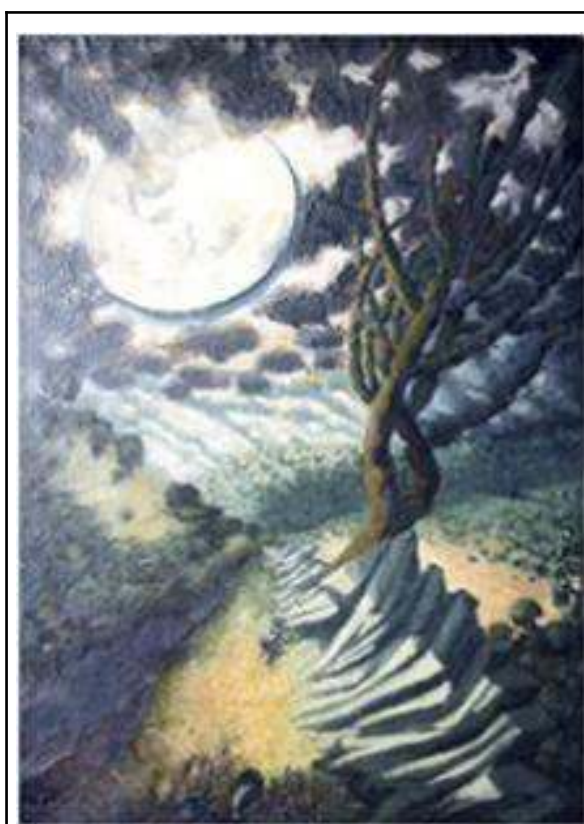

27. Luna, dormilones, osamente, 1956, gravure, Montevideo, coll. part

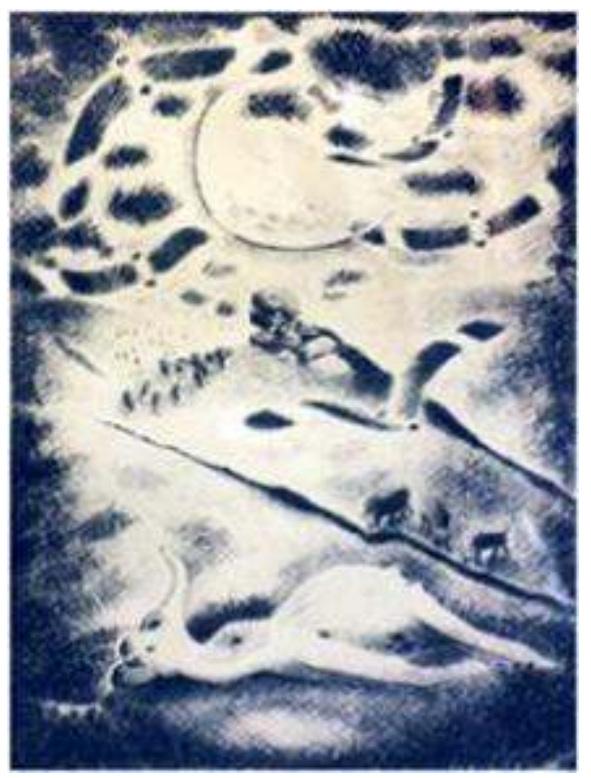

28. Luna de los dormilones, 1950, Montevideo, coll. part 


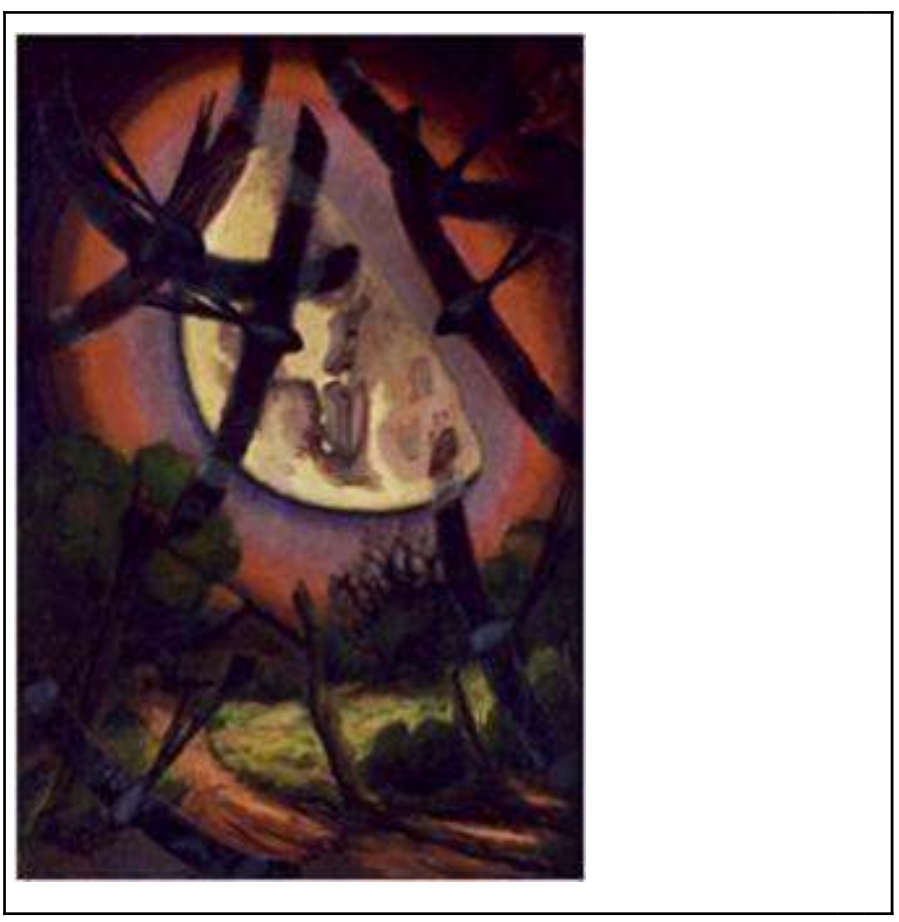




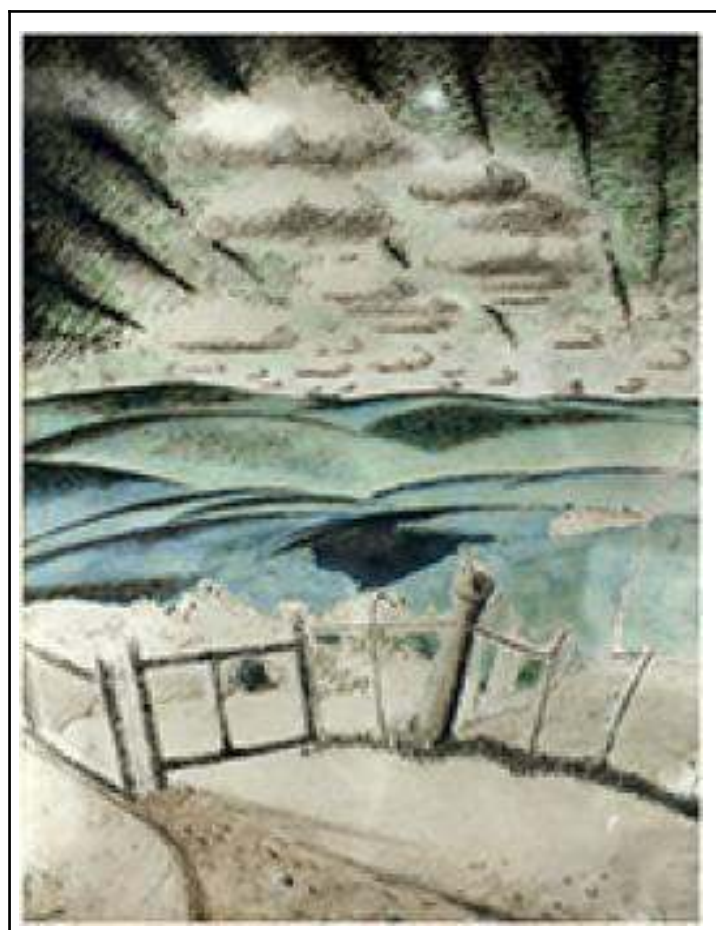

30. Preparando el rodeo,1950, Montevideo, Musée national des Arts visuels

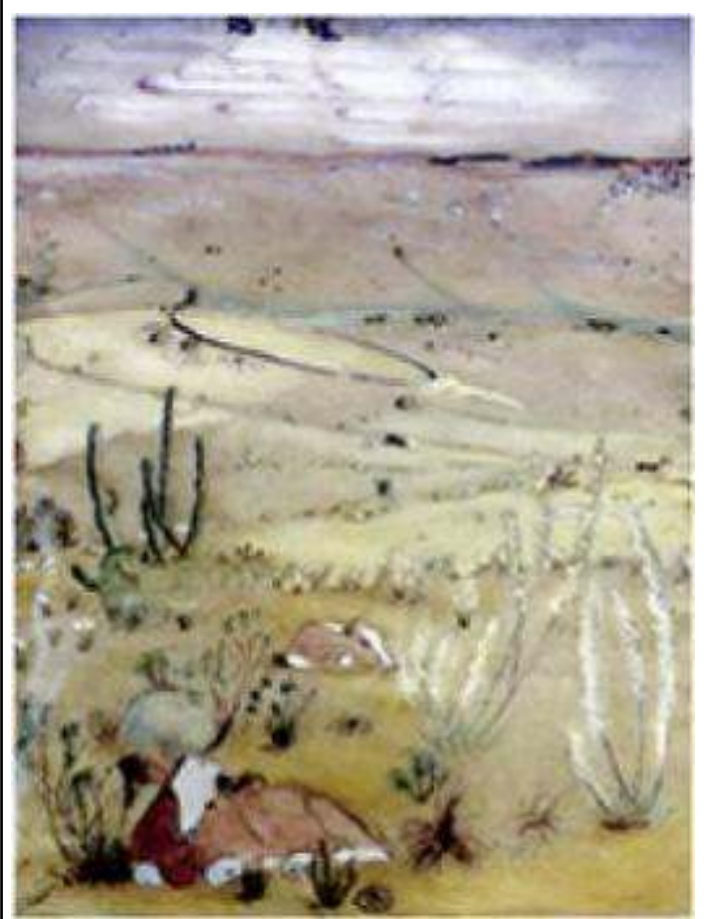

31. Carreta en la estancia, s.d., aquarelle, Montevideo, Musée national des Arts visuels 


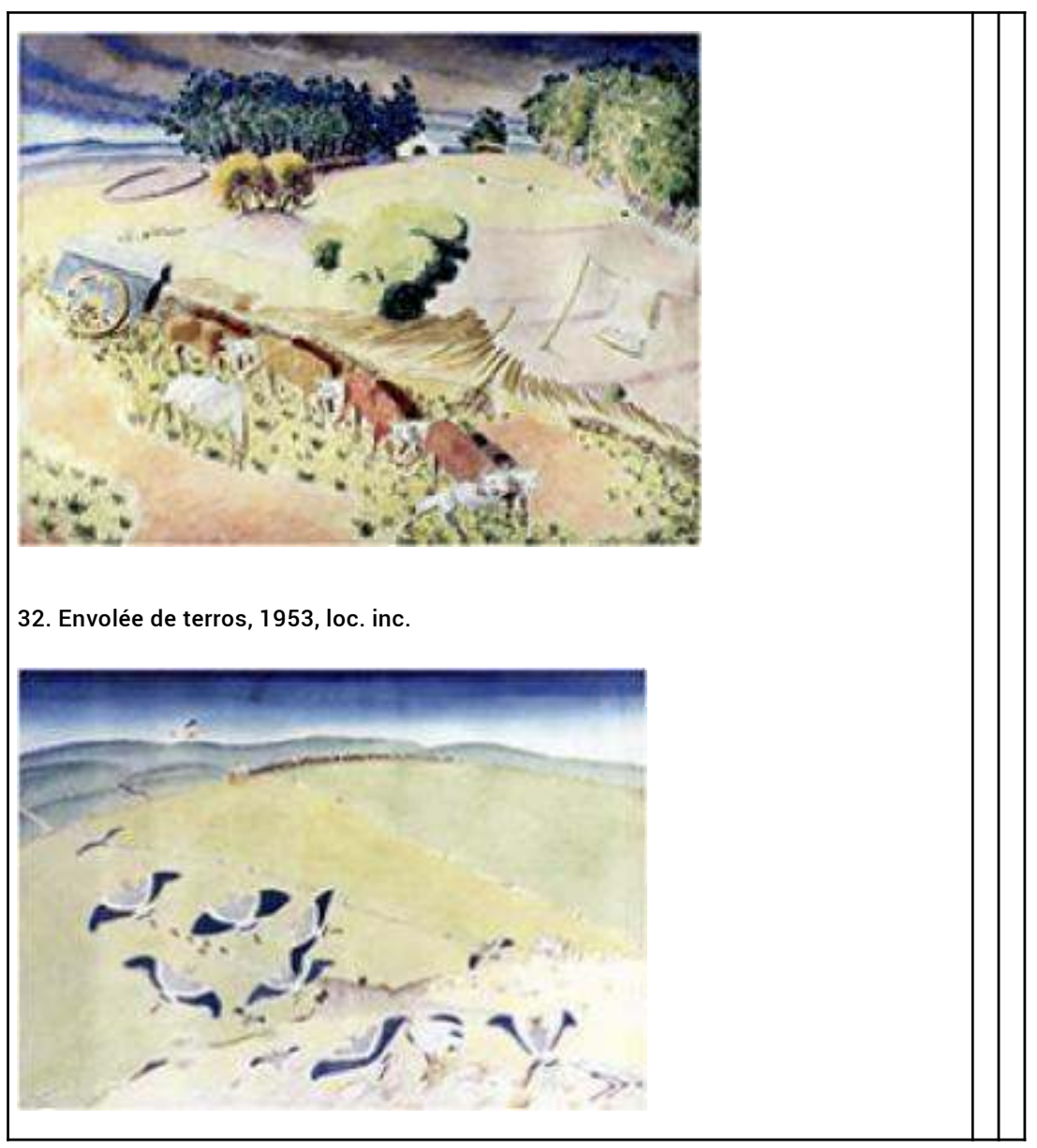

Entre 1957 et 1960, José Cuneo peint à l'huile une série d'œuvres très colorées où dominent les formes circulaires, ovoïdes et serpentines. Le peintre fait ses premiers pas dans l'art abstrait sous le signe du cercle, de l'ovale et de la spirale (cf. ill. 33 à 38). Ces formes font référence à la fermeture sur soi (le cercle), à la fécondité (la forme ovale de l'œuf) et au cycle ovarien au travers du symbolisme de la spirale ${ }^{36}$. Elles symbolisent à la fois la permanence de l'être à travers les fluctuations du changement et le retour à la vie utérine. José Cuneo nous donne inconsciemment la clé de ce symbolisme en adoptant le patronyme maternel Perinetti.

Toutes ces œuvres ne peuvent être qualifiées d'abstraites. En effet, on y rencontre des formes faisant référence à des motifs réels, parfois liés à la thématique du campo, ou dérivant de la série des Lunes. Dans une œuvre datée 1958-6037, le disque jaune traversé par une forme géométrique en zigzag est un rappel du motif lunaire traversé par un nuage effilé de Lune de l'aveugle, Lune du poteau, Lune du ravin ou Lune du peuplier ${ }^{38}$. On retrouve aussi la même composition bâtie sur l'une des diagonales de la toile, mais ici le peintre interdit une lecture orientée dans le sens céleste/terrestre ou terrestre/céleste en signant son œuvre sur plusieurs cotés (d'autres œuvres de cette époque son signées sur deux, trois ou quatre côtés de la toile). Au bout de la trajectoire du zigzag, qui ressemble davantage ici à un éclair, apparaissent deux formes ovoïdes juxtaposées. Ces différents motifs sont entourés d'une succession de croissants de lune de couleur très sombre formant une sorte de réceptacle au milieu duquel se propage une large tache d'un rouge vif. La référence au thème de la fécondation est ici tout à fait explicite. Dans une œuvre de la même période, le peintre fait intervenir des oiseaux très stylisés associés à des formes 
d'escargots et à des ovales dont l'une des extrémités assombrie dessine un croissant de lune ${ }^{39}$. En donnant à la spirale la forme d'un escargot, le peintre fait apparaître l'une des structures les plus fréquentes et les plus essentielles de la nature biologique : «la spirale génératrice ». Apparaît aussi dans ces premières œuvres la forme en point d'interrogation de la Lune aux trois pointes. Parfois, sa base est prolongée pour former une double spirale qui propage son rythme harmonieux sur l'espace de la toile ${ }^{40}$. Une œuvre intitulée Cordon ${ }^{41}$ montre non pas une spirale mais les ondulations d'une forme serpentine de couleur rouge sur un fond gris sur lequel semblent flotter des ovales à l'intérieur tacheté rappelant des molécules et la forme larvaire d'une sorte de batracien. Les formes du monde organique et utérin envahissent progressivement le vocabulaire plastique de Perinetti.

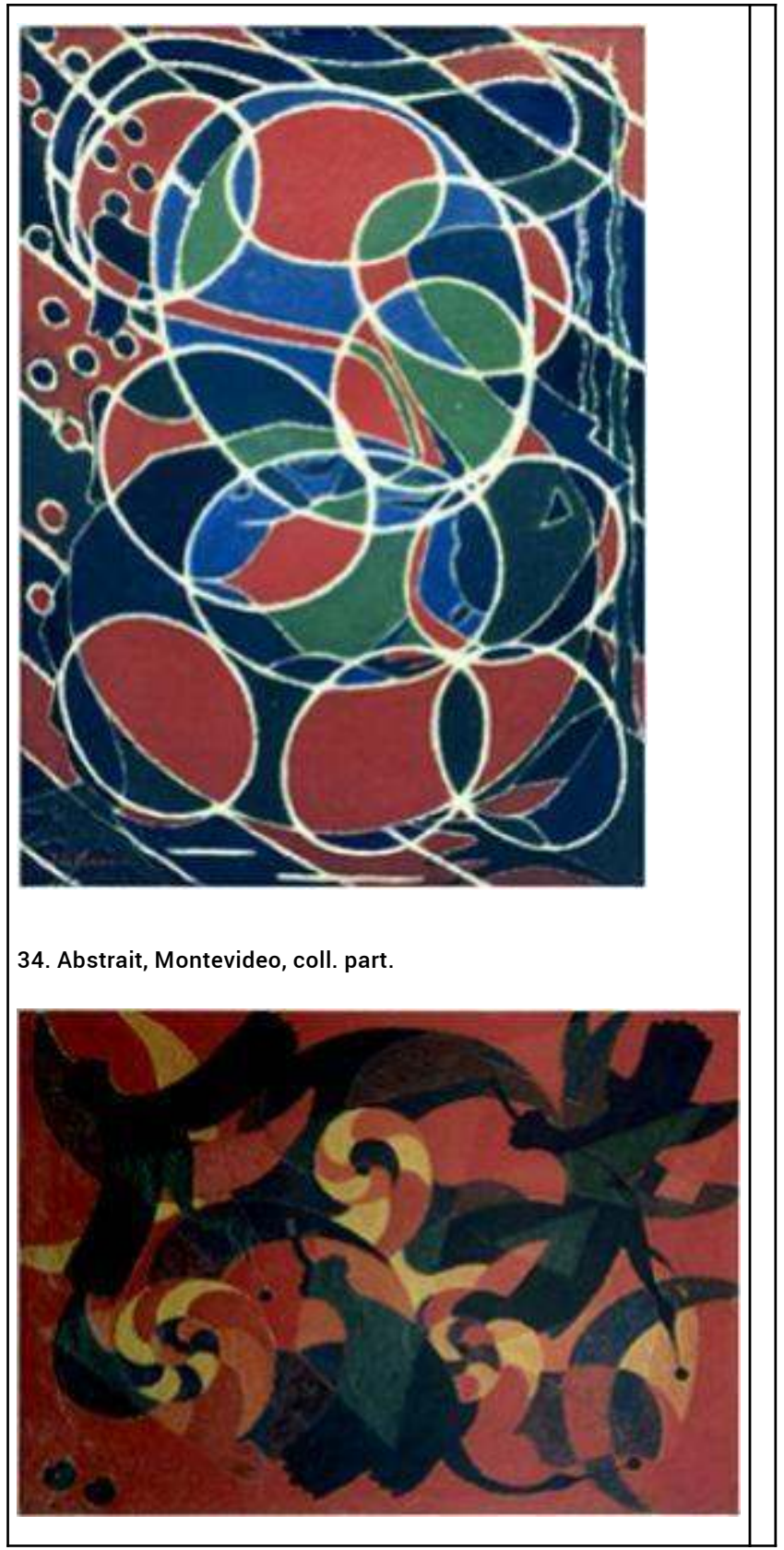


19 Le dynamisme créé par des associations de couleurs inscrites dans des formes géométriques constitue un aspect important de la première période de J. C. Perinetti. Plusieurs œuvres exposées en 1960 à la galerie Montevideo portent des titres faisant référence au mouvement: Rythme nocturne, Bolide, Défilement. Le dynamisme exprimé notamment par la spirale est un des principes dominant de l'esthétique futuriste de Balla et de Boccioni ; il exprime le devenir continuel. Les formes en "allumettes ", que l'on peut voir notamment dans Figures $^{42}$, associées à des cercles, des ovales et à des formes en "parapluie", sont des motifs suspendus dans l'espace comme ceux des mobiles; ils suggèrent le mouvement dans un espace en acte. La composition évoque les stabiles de Calder ${ }^{43}$.

20 Les formes géométriques de ces premières œuvres sont le plus souvent qualifiées par des teintes plates sans effets de matières. Parfois, un espace laissé en réserve sur le fond de la toile les sépare nettement les unes des autres. À partir de 1958, le peintre donne un aspect granuleux à la matière picturale dotant ainsi l'intérieur de ces formes d'un certain relief, et en estompe les contours par des frottis tels que l'on peut en observer dans le ciel de Cerco de tunas (ill. 17) et dans certains paysages du Cerro Largo peints entre 1951 et 1952. À partir de 1960, la matière prend de plus en plus d'importance dans l'œuvre de Perinetti. Le peintre mêle à l'huile différents matériaux, en particulier le sable, la sciure et l'herbe à maté. Les formes serpentines et les spirales acquièrent un puissant relief et une texture que l'on pourrait qualifier d'organique (cf. ill. 35 et 36). La froide géométrie cède la place à un jeu lyrique de formes incarnées dans la matière. Celle-ci déborde des limites de la spirale, se propage sur l'espace de la toile, le gangrène, comme la moisissure sur un mur. Le fond est lui-même auréolé de taches rosâtres semblables à des champignons sur un papier humide ou une peau malade. Parfois le peintre donne à ses formes l'aspect de cuirs tannés, tels ceux que l'on peut voir sécher au soleil dans les estancias ${ }^{44}$. Cependant, la matière est maîtrisée, son usage est, si l'on peut dire, propre, sans bavures, sans provocation d'ordre scatologique, et peut-être est-ce là l'origine même du mépris d'une certaine partie de la critique à l'égard de ces œuvres jugées trop décoratives. L'expansion ou la dilution de la matière engendrent des formes bourgeonnantes et festonnées. Mais la nature n'est-elle pas elle-même productrice de formes semblables? Celles justement qu'observait Léonard de Vinci sur les murs décrépis, les cendres du foyer, les nuages ou la boue et dans lesquelles il voyait des " paysages divins ». Interviennent de plus en plus, après 1960, les formes hélicoïdales, embryonnaires, fœtales, des serpentins gonflés comme des intestins. Le peintre fait intervenir pour la première fois dans son œuvre l'animal emblématique de la pampa, la mulita (sorte de priodonte ou de tatou), étrange survivant des temps préhistoriques. L'espace oblong, dans lequel apparaissait la lune de Lune de la caverne $e^{45}$, est devenu la trompe utérine abritant la forme tentaculaire d'une sorte d'animal en gestation ${ }^{46}$. Tandis que plane encore dans les œuvres de Perinetti la sombre envergure des engoulevents, les céphalopodes font leur apparition dans ce monde utérin et signalent l'avènement de l'élément aquatique dans l'œuvre de Cuneo. Parfois, surgit sur le fond neutre du papier un motif isolé appartenant à son iconographie figurative, mais métamorphosé par la pulsion du geste instinctif (bien que contrôlé). Nous voyons apparaître alors au travers des coulées de l'encre noire et des griffures de la plume, la silhouette barbelée des clôtures du campo. Masques primitifs, silhouettes zoomorphes et anthropomorphes grouillent enfin dans une jungle de formes enchevêtrées. On pourrait continuer longtemps à faire l'inventaire des formes qui peuplent les œuvres de Perinetti; plus encore que les cieux nocturnes des paysages 
cunéens, ces œuvres offrent un vocabulaire plastique infini, à l'image de celui que produit la nature mais aussi de celui que font surgir l'inconscient et le rêve.

\section{Noche, s.d., Montevideo, Musée national des Arts visuels}

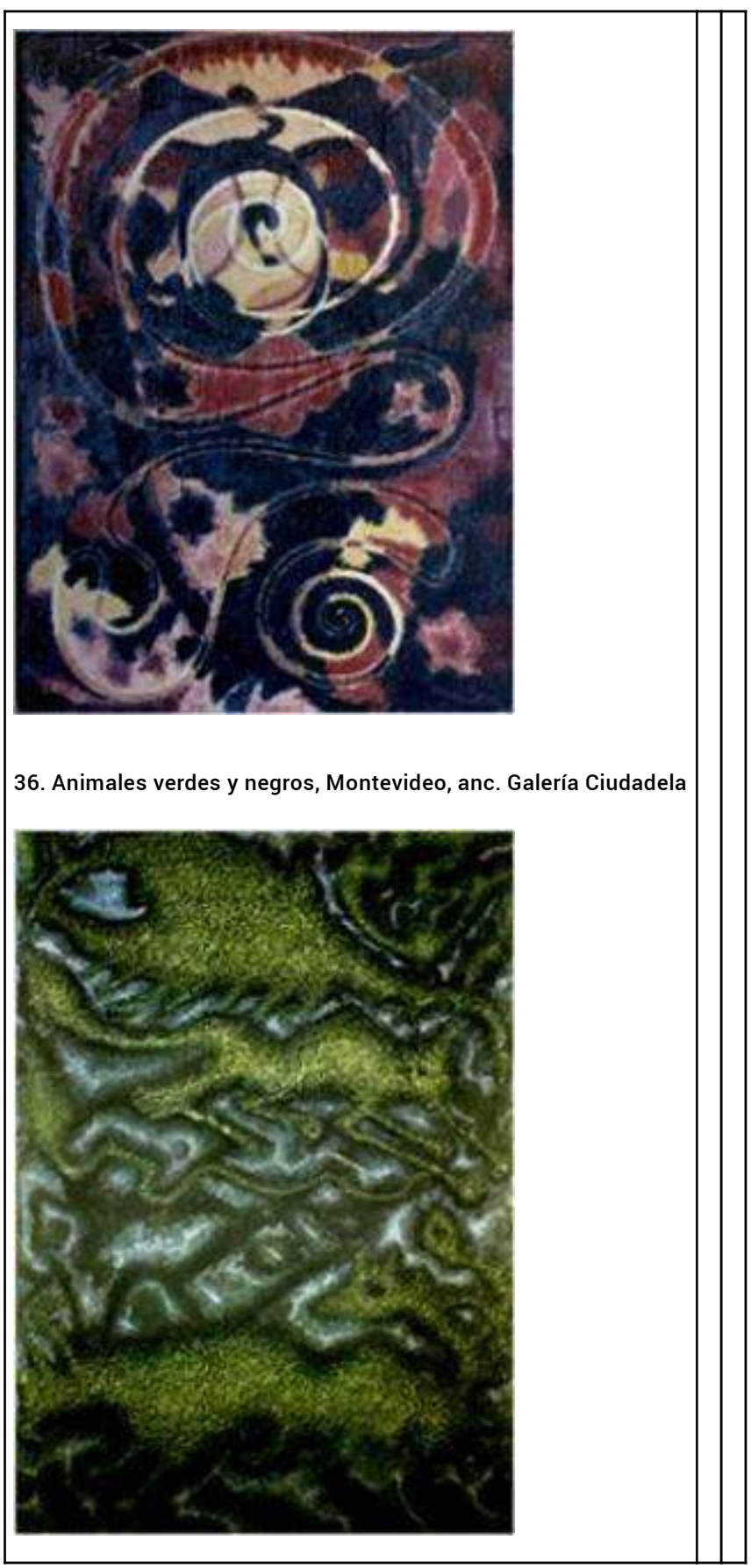

L'évolution relativement brusque de l'œuvre de Perinetti à partir de 1960 peut être, dans une certaine mesure, comparée au passage du planisme à «l'expressionnisme» dans l'œuvre de Cuneo, et cela tant du point de vue de la couleur que de la forme. Les couleurs pures des années 1957-1959 cèdent la place à des tons plus sombres ou confrontés à la matière noire. À partir de 1960, la nuit se propage dans l'œuvre de Perinetti comme elle a surgi en 1930 dans l'œuvre de Cuneo. Jusqu'au début des années soixante-dix, la couleur 
est aussi variable dans l'œuvre de Perinetti que les formes qu'elle qualifie et que le médium au travers duquel ces formes prennent vie: huile, matières diverses, pastel, aquarelle, encre. Chaque œuvre témoigne d'une expérimentation nouvelle de la couleur et de la forme. Dans les œuvres de Perinetti peintes à partir de 1960, l'espace est conçu selon un principe stratigraphique qui se manifeste par la superposition de couches de matières ou de réseaux de lignes et de formes. C'est en quelque sorte la conceptualisation du phénomène de superposition et de recoupement produit par les ailes des engoulevents sur le réseau obscur des nuages dans Lune du poteau et Lune du ravin. Cette utilisation de réseaux superposés n'est peut-être pas sans rapport avec la conception du mouvement dans l'art cinétique, pour lequel José Cuneo a manifesté un certain intérêt dans les années soixante. Mais le peintre uruguayen n'a pas recours, comme Vasarely, à des effets $\mathrm{d}^{\prime}$ «illusion psycho-physiologiques». Perinetti ne fait que confirmer la conception spatiale élaborée par Cuneo dans la série des Lunes. Le problème du rapport de la forme et du fond a hanté Cuneo tout autant que Perinetti, mais ce dernier le résout dans un vocabulaire abstrait. Par ailleurs, on peut observer que l'obscurcissement des zones périphériques de la toile qui crée dans les paysages figuratifs un lien optique entre la terre et le ciel s'est intensifié, opacifié; ces zones forment maintenant une structure infranchissable, qui cerne la totalité de l'espace désormais refermé sur lui-même: l'espace pictural revendique librement son autonomie absolue ${ }^{47}$.

Bien que José Cuneo ait manifesté à partir d'une certaine époque un grand intérêt pour l'art non figuratif ou abstrait, en particulier pour le groupe Cobra, mais aussi pour Jean Dubuffet et l'art informel, les œuvres qu'il peint pendant les vingt dernières années de sa vie ne reflètent l'influence directe d'aucune de ces tendances de l'art contemporain. Karel Appel écrit : « L'acte de création a lui-même beaucoup plus d'importance que l'objet créé, et ce dernier gagne en signification dans la mesure où il porte les traces du labeur qui l'a engendré et n'est point parachevé ${ }^{48}$. " J.C. Perinetti n'avalise pas une telle conception de l'acte créatif. Ses œuvres sont des objets travaillés et finis. Ce sont des œuvres de vieillesse ou, si l'on préfère, de maturité, qui reflètent à la fois une extrême vitalité, une grande maîtrise des différentes techniques picturales (huile, aquarelle, encre et pastel), en même temps qu'une sorte de régression, non pas d'ordre esthétique mais intime, psychologique, régression dont le peintre, avant tout soucieux de justifier ses options formelles et la modernité de son langage plastique, n'a pas été, semble-t-il, lui-même conscient. Régression qu'il n'a pas voulu revendiquer ouvertement, peut-être aussi pour ne pas tenter la critique de dévier vers une interprétation qui aurait tenu compte de facteurs autres que formalistes. Pour la première fois, le peintre exprime son moi et non l'imaginaire collectif; l'origine de l'œuvre de Perinetti est à chercher avant tout dans l'œuvre de José Cuneo et dans l'inconscient de l'artiste. 
37. Mar, s.d., Montevideo, Musée national des Arts visuels

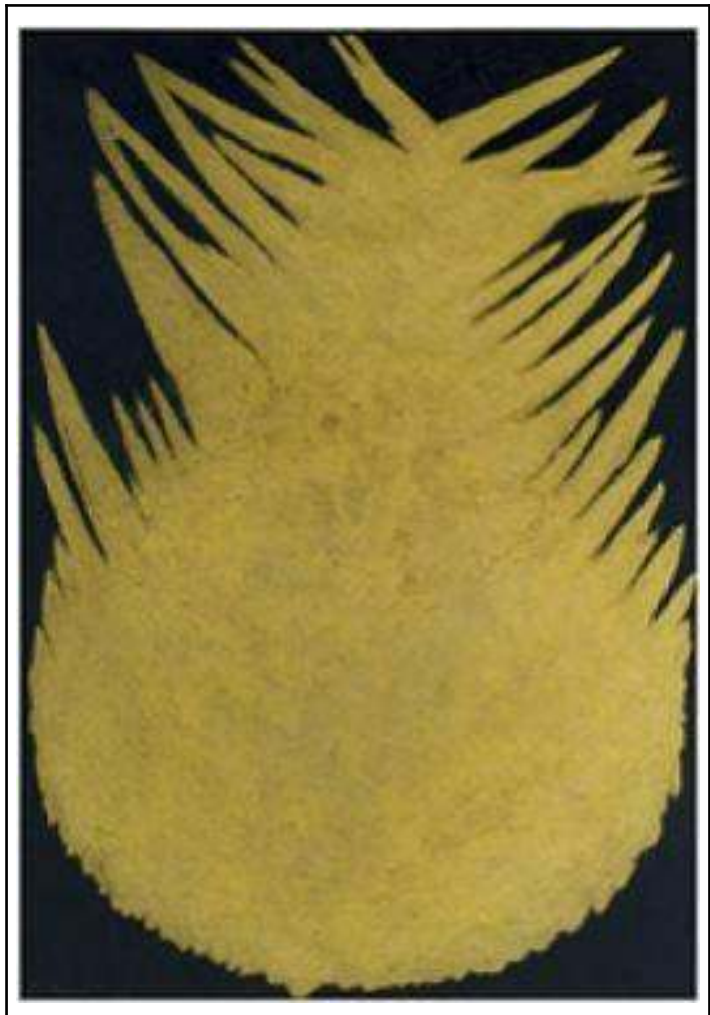

38. Abstrait, s.d.,. Montevideo, anc Galería Ciudadela

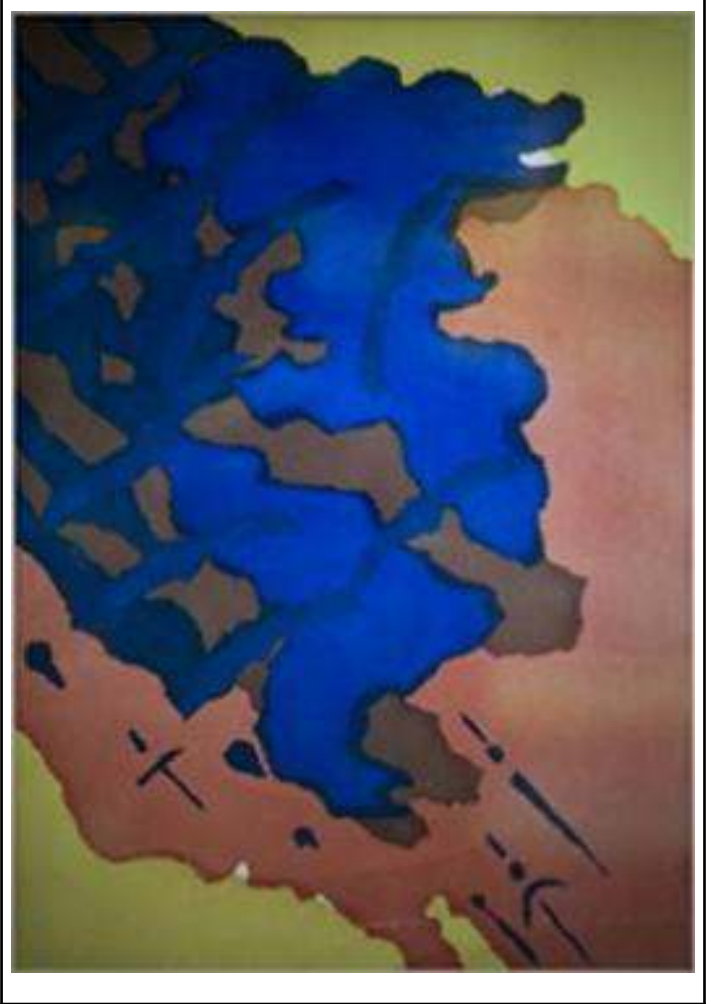




\section{BIBLIOGRAPHIE}

APPEL Karel, cité dans Dictionnaire général du surréalisme et ses environs, Paris, PUF, 1982.

ARGUL José Pedro, José Cuneo paisajista del Uruguay, Montevideo, San Felipe y Santiago, 1949.

CARDIs Régine, José Cuneo Perinetti, peintre uruguayen - monographie et catalogue raisonné, Thèse de doctorat, Université de Toulouse-le-Mirail, 1998.

CUNEo José, « Cuneo, la avidez de los 84 años » (entretien), in Marcha, Montevideo, 19 décembre 1971.

CUNEO José, «El maestro de las lunas explica porque cambio de nombre y de lenguaje » (entretien), in El País de los Domingos, Montevideo, septembre 1975.

CUNEO José, « La obra de Barradas, Estudio intentado desde el punto de vista de un pintor », El Día (Montevideo), 22 octobre 1930.

CUNEO José, « Pintura abstracta », in Esfuerzo, Montevideo, n³7, 1958.

DANTO Arthur, L'Assujettissement philosophique de l'art, Paris, Le Seuil, 1993. traduction française de l'édition américaine, New York, Columbia University Press 1986

DIESTE Eduardo, « Discusion estética y ejemplos », in Teseo, Montevideo, 1925, p. 74.

LHOTE André, Traité du paysage, Paris, Floury, 1939.

PELUfFo linARI Gabriel, « Pedro Figari et le nativisme du Rio de la Plata », in catalogue de l'exposition Pedro Figari, Paris, Pavillon des Arts, 1992, pp. 55 à 73.

PEREDA Raquel, José Cuneo, retrato de un pintor, Montevideo, Ed. Galería Latina, 1988.

SOURIAU Étienne, Vocabulaire d'esthétique, Paris, PUF, 1990.

VITUREIRA Cipriano, « Los paisajes lunares de Cuneo », in La Mañana, Montevideo, 25 septembre 1936.

\section{NOTES}

1. José Cuneo obtient en 1953 le Grand Prix national de peinture à la première Biennale nationale d'arts plastiques de Montevideo, qui consiste en une bourse d'études de deux ans en Europe. En créant cette Biennale, accessible uniquement aux lauréats du Grand Prix du Salon National, le ministre de l'Instruction publique, Justino Zavala Muniz, entend donner à ces artistes une opportunité nouvelle de renouvellement au contact de l'art européen. Avant cette date, le peintre a effectué six séjours en Europe (France et Italie) : 1907/1909, 1912, 1916/1917, 1925, 1927/1930, 1938/1939 (cf. CARDIS : 1998).

2. Texte dactylographié, archives Cuneo (cf. CARDIS : 1998, p. 119).

3. Le texte de cette conférence a été publié dans le journal Esfuerzo en 1958 (CUNEO : 1958, p. 10).

4. «Si mi pintura abstracta tiene interés, se debe, antes que a toda otra consideración, a mi convencimiento, a mi fé absoluta, inconmovible, de que existe un nuevo lenguaje de la pintura de nuestro tiempo. Este lenguaje me ha costado y me cuesta dominar, exactamente coma el hablado 
a toda persona que se somete a estudiar un nuevo idioma " (texte dactylographié, archives Cuneo ; cf. CARDIS : 1998, p. 208).

5. La lettre que José Cuneo adresse à Pierre Cabanne en 1962, dans laquelle il affirme la supériorité de l'« École de Paris ", en est un des derniers témoignages (cf. CARDIS : 1998, p. 208).

6. Entre 1907 et 1909, José Cuneo étudie la peinture à Turin, dans l'atelier d'Anton Mucchi. En 1911, il retourne en Italie, près de Rome, où il peint en compagnie de Mucchi des paysages du lac d'Albano, de Nemi et les jardins des résidences d'été. En 1912, il suit les classes de dessin du peintre espagnol Anglada Camarassa et les classes de peinture de Kees Van Dongen à l'Académie Viti ; il visite la Belgique (Ostende, Liège, Bruges, Gand, Malines, Anvers) et passe un mois à Londres. Entre 1916 et 1917, il séjourne pendant une dizaine de mois à Paris. En 1925, il visite l'Exposition internationale des arts décoratifs à Paris ainsi que de nombreux musées et expositions d'arts décoratifs, en Allemagne (Hambourg, Berlin, Postdam) et en Italie (Turin, Milan, Florence, Monza). Lauréat du concours national de peinture de 1926, il part en France deux ans plus tard et va peindre à Cagnes-sur-Mer jusqu'en 1930. En 1938, José Cuneo se rend en France et en Italie, où il peint à l'aquarelle une série de paysages de Venise (cf. CARDIS : 1998, pp. 21, 22, 69).

7. Pedro Blanes Viale, né à Mercedes (Uruguay) en 1879 et décédé en 1926 à Montevideo, a étudié la peinture à l'Académie de San Fernando à Madrid et, quelques années plus tard, dans l'atelier de Benjamin Constant et dans celui d'Antonio de La Gándara à Paris.

8. Selon l'historien de l'art uruguayen José Pedro Argul, les œuvres du peintre turinois Antonio Fontanessi (1818-1882), que José Cuneo a pu voir au Palais royal et au Musée civique de Turin, sont à l'origine de la vocation de paysagiste du peintre uruguayen (ARGUL : 1949, p. 9).

9. José Cuneon cité par PEREDA : 1988, p. 15.

10. Ibid., p. 2I. L'œuvre d'Anglada Camarassa a connu un vif succès en Amérique latine. Parmi ses plus fervents admirateurs figuraient, notamment, Pedro Figari, et des artistes de la génération de José Cuneo, Etchebarne Bidart, Carmelo de Arzadun, Manuel Rosé, qui se sont formés dans l'atelier de l'artiste espagnol à Paris.

11. «Islas »: bois d'eucalyptus plantés en lignes régulières, isolés dans la pampa, et destinés à protéger le bétail par grand vent. On peut voir ce type de bois dans La Aguada, (ill. 6).

12. Le critique uruguayen Eduardo Dieste appelle " planisme » un style particulier de la peinture uruguayenne des années vingt, qui cumule divers aspects de la peinture française postimpressionniste et se caractérise surtout par une volonté constructive et l'utilisation de couleurs primaires et secondaires disposées en aplats. Les principaux représentants de ce style sont José Cuneo, qui expose pour la première fois des œuvres pIanistes en 1918 à Montevideo, Carmelo de Arzadun, Humberto Causa, Domingo Bazurro, Guillermo Rodríguez, Alfredo Sollazo, Melchor Méndez Magarinos et Petrona Viera.

13. La Tour de la vigie (Maldonado), loc. inc. reprod. dans PEREDA : 1988, p.57

14. DIESTE :1925, p. 74.

15. Nous ignorons dans quelles circonstances le peintre uruguayen a pu voir des œuvres de Soutine : à Cagnes-sur-Mer, où il séjourne entre 1928 et 1930, ou à Paris. La première exposition particulière de Soutine a eu lieu en 1927 à Paris et la première monographie, écrite par Waldemar George, a été publiée l'année suivante. Avant de se rendre à Cagnes-sur-Mer, José Cuneo n'a probablement eu connaissance ni de l'une ni de l'autre. La confrontation des paysages de Cagnes peints par José Cuneo avec des paysages de Soutine représentant les mêmes sites nous permet cependant d'affirmer que Cuneo s'est directement inspiré de certaines œuvres du peintre russe (cf. CARDIS : 1998, p. 69).

16. José Cuneo, cité par PEREDA : 1988, p. 37.

17. VITUREIRA : 1936, p. 1.

18. L’Escuela Taller de Artes Plásticas (E.T.A.P.) fondée à Montevideo en 1933, présidée par José Cuneo, a pour but de réunir les peintres et les sculpteurs isolés qui souhaitent défendre leur 
statut d'artiste. Elle offre un local aux artistes formés en Europe désireux de faire partager leur expérience à travers des discussions, des conférences ou des ateliers. Joaquín Torres García donne des conférences à l'E.T.A.P. à son retour en Uruguay en 1934.

19. LHOTE : 1939, p. 157. Dans un entretien pour l'hebdomadaire Marcha, José Cuneo paraphrase André Lhote : «En el 800 había muchos pintores que hacían la figura, y dejaban el fondo neutro, inexpresivo. Quedaban espacios huecos. En la pintura actual, el cuadro tiene que estar lleno, de arriba abajo y no puede haber vacíos. No quiere decir que yo lo consiga siempre. A veces logro esa consustanciación de fondo y forma » (Marcha, 1971, p. 30).

20. Lune de l'herborisatrice (Luna de la Yuyera), Montevideo, coll. part, reprod. dans PEREDA : 1988, p. 124.

21. Lune noire, Mains pattes, Oiseau, Montevideo, Musée national des Arts visuels (Montevideo).

22. Le "nativismo » est la culmination esthétique et philosophique du "criollismo ", incarné dans l'épopée gauchesque du Martín Fierro de José Hemández (1872) et dans les gauchos peints par Juan Manuel Blanes (Montevideo, 1830-1901). Les principaux représentants du "nativismo » sont le peintre Pedro Figari, le compositeur Fabini et les poètes Fernan Silva Valdés (auteur de Agua del Tiempo. Poemas Nativos, 1921) et Pedro Leandro Ipuche (le premier à avoir employé ce vocable en 1916 dans La Pajarera nativa).

23. PELUFFO LINARI : 1992.

24. L'avocat Pedro Figari (Montevideo, 1861-1938) a commencé sa carrière de peintre à l'âge de soixante-deux ans. Il est l'auteur, notamment, de "Arte, estética e ideal », publié en 1912 à Montevideo.

25. Le terme de baroque est employé ici en tant que catégorie stylistique trans-historique (cf. SOURIAU : 1990, p. 226).

26. El País de los Domingos, 1975.

27. Cette étude se résume à une seule page dactylographiée et paraît inachevée. Aucune version manuscrite n'a été retrouvée (cf. CARDIS : 1998, p. 208).

28. Manuel B. Cossio, El Greco, Madrid, Suárez, 1908 ; J. F. Willumsen, La jeunesse du peintre El Greco, Paris, Crès, 1927, 2 vol.

29. CUNEO : 1930.

30. Rafael Barradas (Montevideo, 1890-1929) a passé la majeure partie de sa vie en Espagne.

31. Le fonds Cuneo de la bibliothèque du MNAV compte un exemplaire de la correspondance de Gauguin annotée par José Cuneo. Dans son article sur Rafael Barradas, José Cuneo évoque longuement le cas de Gauguin pour expliquer la différence qu'il entend entre "personnalité individuelle » et « personnalité raciale » (CUNEO : 1930).

32. CUNEO : 1930.

33. PEREDA : 1988 , p. 35.

34. Pedro Figari a lui-même été surnommé par la critique française le « Gauguin uruguayen ».

35. Lune des trois pointes, Paris, Centre Georges Pompidou, fonds du musée du Jeu de Paume.

36. La spirale apparaît sur les idoles féminines de l'art paléolithique.

37. Lunes, Montevideo, coll. partic.

38. Lune de l'aveugle, Montevideo, coll. partic.; Lune du poteau, loc. inc., connue d'après photographies; Lune du ravin, loc. inc., Montevideo, vente Gomensoro, 31 mai 1993, n 76 du catalogue ; Lune du peuplier, Montevideo, coll. part.

39. Sans titre, Montevideo, succession José Pedro Argul.

40. «Forme mathématique idéale, par les passages insensibles et continus qu'elle ménage, par des modulations de couleurs d'une aire à l'autre, la spirale (et surtout la double spirale) permet à de nombreux plasticiens (notamment Johannes Itten, peintre du Bauhaus, Moholy-Nagy, Max Bill) des recherches sur la couleur et l'espace. L'harmonieuse perfection de sa courbe qui se déroule dans le Temps diffuse une charge lyrique à ce qui pourrait ne ressortir qu'à une froide géométrie » (SOURIAU : 1990). 
41. Cordon, Musée de Maldonado (Uruguay).

42. Figures, loc. inc. Montevideo, vente Castells, 28 août 1997, n 43 du catalogue.

43. Calder est l'artiste nord-américain que José Cuneo appréciait le plus (CARDIS : 1998, p. 27, note 30 ).

44. Cuir, Montevideo, coll. partic.

45. Lune de la caverne, connue d'après photographies.

46. Mains pattes, 1961, Montevideo, MNAV.

47. Vers 1975, le peintre revient à une iconographie plus figurative. Il exécute au pastel gras et sur papier de verre une série d'œuvres de petites dimensions qui représentent des animaux typiques de la faune uruguayenne, le plus souvent des oiseaux. Le peintre signale son retour à la figuration en signant la plupart de ces œuvres du double non de Cuneo Perinetti (cf. CARDIS : 1998, p. 142).

48. APPEL Karel : cité dans Dictionnaire général du surréalisme et ses environs, Paris, PUF, 1982, p.354

\section{RÉSUMÉS}

C'est dans la peinture de paysage que José Cuneo (1887-1977) projette l'essentiel de ses ambitions esthétiques. Il peint la campagne uruguayenne dans un style appelé "planismo ", qui tente d'assumer l'héritage coloriste de la peinture impressionniste et postimpressionniste, ainsi que l'orientation constructive et formelle des héritiers de Cézanne. La découverte de l'œuvre de Soutine l'oriente vers une expression qui prend en compte le mouvement et la libre interprétation du monde visible. Dans la série des « Ranchos y Lunas », il adapte une conception spatiale héritée de Soutine à une vision panthéiste et métaphorique du paysage local. Âgé de 70 ans, Cuneo-Perinetti expérimente l'abstraction en procédant à l'examen rétrospectif de sa propre évolution affective et mentale.

José Cuneo (1887-1977) proyecta en la pintura de paisaje lo esencial de sus ambiciones estéticas. Pinta el campo uruguayo en un estilo llamado "planismo » donde intenta plasmar la herencia colorista de la pintura impresionista y post-impresionista y la orientación contructiva y formal de los discípulos de Cezanne. Descubriendo la obra de Soutine, Cuneo adopta una expresión que se apropia el movimiento e interpreta libremente el mundo visible. En la serie de los "Ranchos y Lunas" adapta una concepción espacial heredada de Soutine con su visión panteísta y metafórica del paisaje local. Luego, en los años 70 Cuneo experimenta la abstracción entablando un proceso de evaluación de su propia evolución afectiva y mental.

\section{INDEX}

Palabras claves : Uruguay, Cuneo, arte moderno, paisaje, nativismo, expresionismo, abstracción Mots-clés : Uruguay, Cuneo, art moderne, paysage, nativisme, expressionisme, abstraction 
AUTEUR

RÉGINE CARDIS

docteur en histoire de l'art. regine.cardis[at]yahoo.fr 